\title{
A STUDY OF THE CURRENT TRANSFORMER WITH PARTICULAR REFERENCE TO IRON LOSS
}

\author{
By P. G. Agnew
}

\section{CONTENTS}

2. Methods for determining ratio and phase angle

4. Conditions for linear ratio and phase-angle curves. . . . . . . . . . . . . . . . 439

5. Exponents and the ratio of variation in iron losses . . . . . . . . . . . 442

6. Ratio of variation and slope of ratio curve . . . . . . . . . . . . . 457

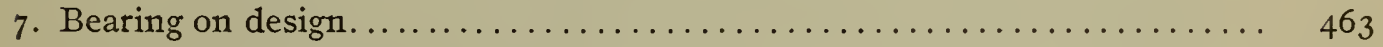

8. Distortion of wave form. .......................... 464

9. Effect of wave form on the ratio and phase angle............... 470

ro. Summary.................................. 472

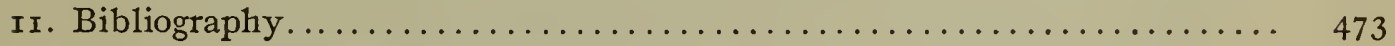

\section{INTRODUCTION}

In a theoretically perfect current transformer the currents would have a ratio equal to the inverse ratio of turns, and the secondary current would be exactly opposite in phase to the primary current. This ideal condition is shown in Fig. I, where $n$ is the ratio of turns, and $I_{1}$ and $I_{2}$ are the primary and secondary currents, respectively.

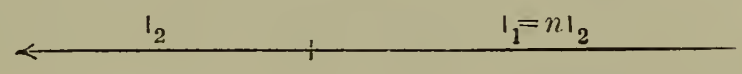

Fig. 1.- Vector diagram of an ideal current transformer.

In the actual transformer neither of these conditions is realized, since an appreciable part of the primary current is required to excite the core. Hence, the ratio of the primary to the secondary current is greater than the inverse ratio of turns, and the two currents are not quite in phase but differ by a small angle. (For 
the case of a leading current in the secondary circuit the ratio may be less than the ratio of turns, but this is a condition which is never met in practice.) A further complication is introduced by the fact that the flux density, and therefore the core loss and magnetizing current are functions of the current, so that in general, both ratio and phase angle are different for different values of the current load. Moreover, the impedance of the instruments connected with the secondary determine, in part, the value of the flux, so that the whole ratio-current curve may be changed by an increase or decrease in the impedance connected with the secondary.

Another possible disturbing factor is wave distortion in the transformer, but it will be shown later that this is entirely inappreciable under practical conditions.

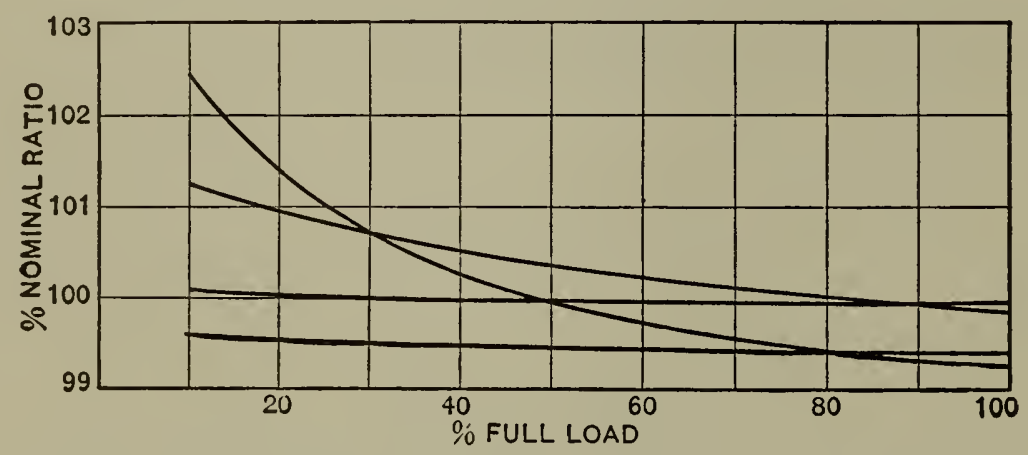

Fig. 2.-Typical ratio curves.

Fig. 2 shows some typical forms of the ratio-current curves of current transformers, the ordinates being the ratios (primary to secondary) expressed in per cent of nominal values. Fig. 3 shows a few typical phase angle curves, the ordinates being the angles by which the reversed secondary current leads the primary. These curves as well as those for the ratios are plotted from actually determined values. ${ }^{1}$

It will be seen that both the ratio and the phase angle curves tend to become horizontal straight lines near full load, and that they rise more rapidly toward the low current end of the curve.

\footnotetext{
${ }^{1}$ For a considerable number of such curves, giving numerical values, connected load, etc., sec:

This Bulletin, 6, p, 298, r909, Reprint No. I30.

L. T. Robinson: Trans. Am. Inst. E. E., 28, p. 1005; I909.
} 
It is an interesting fact that both types of curves should be so very similar in their general shape.

Although during a considerable experience in the testing of transformers it had been noticed that many transformers showed a tendency to turn down instead of up at the extreme low current end of the ratio curve, these cases had been passed over with the idea that they were probably due to inaccuracies of measurement, since the difficulties in making measurements of high accuracy at currents below about 20 per cent of full load are very great. However, Fig. 4 shows the 25 and 60 cycle ratio curves of a transformer exhibiting unusual and remarkable characteristics. It will be seen that at 60 cycles with the small impedance

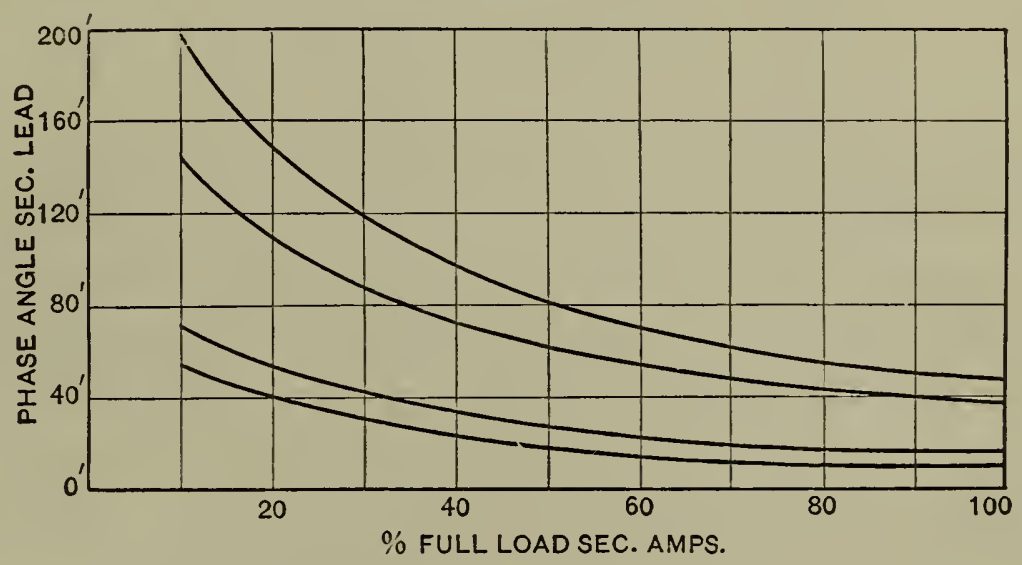

Fig. 3.-Typical phase angIe curves.

load used, the ratio increases with increasing current throughout the entire range from 5 per cent of rated load to full load. At 25 cycles the ratio increases with increasing current from the value at low current load to a maximum at approximately half load and then gradually decreases to full load.

In the 60 cycle curve the total change in the ratio from ro per cent to full load is 0.23 per cent, a quantity about Io times as great as the sensibility of the method used, if averaged over the whole range. The experimental evidence that the 25 cycle curve passed through a maximum was equally good. The direct determinations are indicated by circles all lying on the curves in Fig. 4. The points indicated by crosses are computed values which will be discussed later. 
Since these measurements were made Edgcumbe has described a transformer showing a maximum in its ratio curve, but otherwise such an anomalous behavior did not seem to have been observed, and it is generally considered by engineers that the ratio always decreases with increasing current. Edgcumbe gave

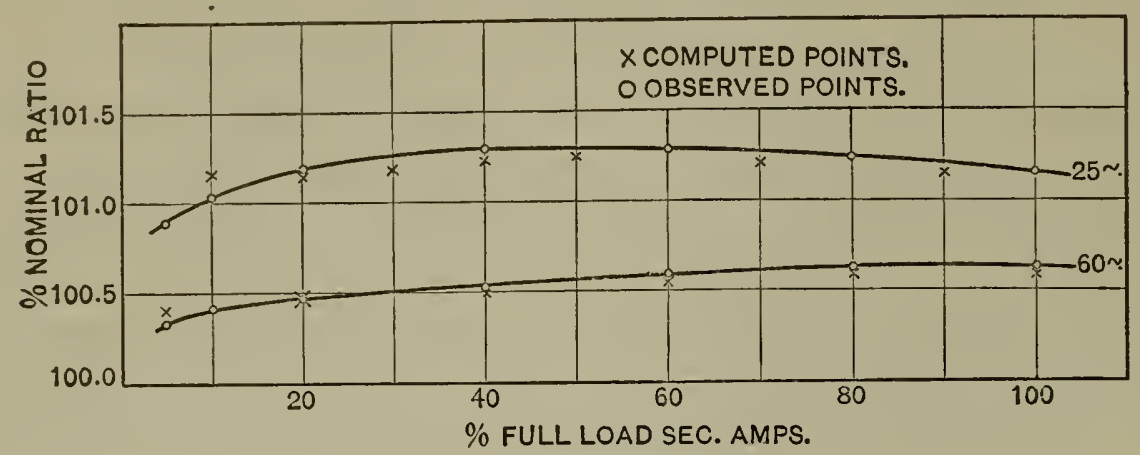

Fig. 4.-Ratio curves of Transformer $F$.

no theoretical explanation of the anomaly. ${ }^{2}$ It therefore seemed important to determine if possible whether there might be theoretical errors in the method of measurement used, or, if not, whether some light might not be thrown upon the nature of the iron losses at such low flux densities as are used in current trans-

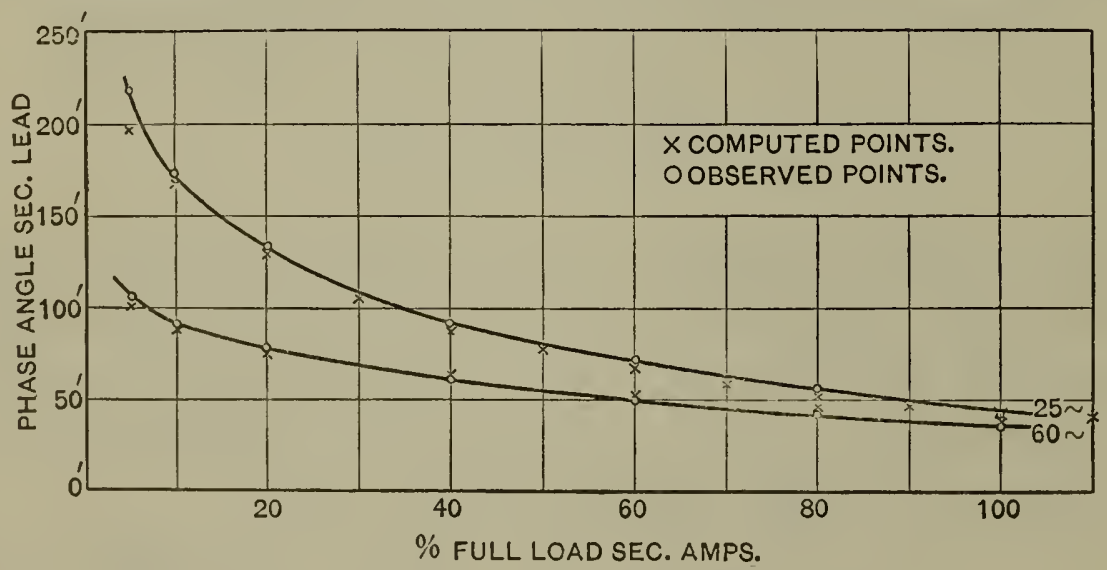

Fig. 5.-Phase angle curves of transformer $F$.

formers, and upon their possible effect on the ratio and phase angle of the instruments.

Many other questions in regard to the behavior of the current transformer are involved, such as: the agreement between theory and practice; possible distortion introduced by the transformer

${ }^{2}$ Iilec. Rev. Lond., 67, p. I63; I910. 
itself; the bearing of this upon the definitions of ratio and phase angle involved in different methods of measurement; and the effect of wave form upon ratio. These were necessarily considered in connection with this investigation, as well as with other transformers.

\section{METHODS FOR DETERMINING RATIO AND PHASE ANGLE}

The most accurate methods for the measurement of ratio and phase angle are null methods depending upon the potentiometer principle. The electromotive forces at the terminals of two noninductive shunts placed in the primary and secondary, respectively, are opposed; and the resistance of the secondary shunt is

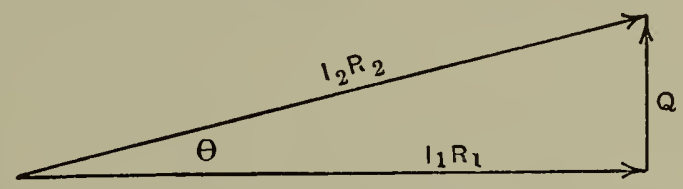

Fig. 6. - Vector relations in method of measuring ratio and phase angle.

adjusted until the in-phase component of the resulting electromotive force is zero. The current relations are shown in Fig. 6. where $I_{1} R_{1}$ and $I_{2} R_{2}$ are the electromotive forces, $\theta$ the phase angle, and $Q$ the quadrature component of the resultant electromotive force. The method in use at the Bureau of Standards is

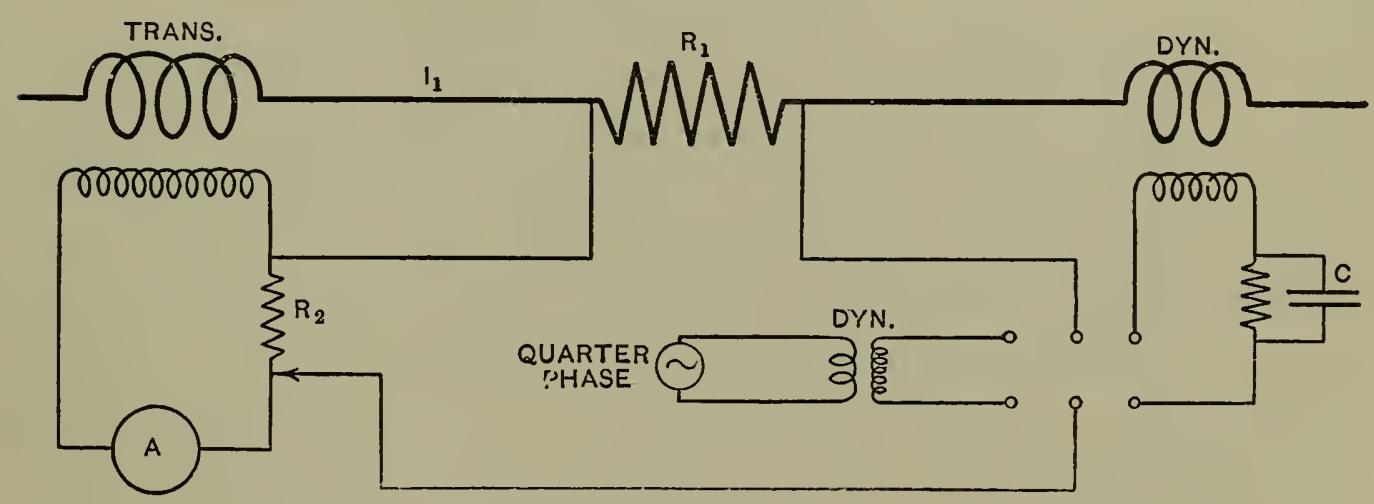

Fig. 7.-Connections for measuring ratis and phase angle,

to place the series coil of an electrodynamometer in series with the primary shunt, and use the moving coil as a detector for setting the in-phase component of the resultant at zero. The complete arrangement is shown in Fig. 7. The switch is thrown to the right and $R_{2}$ adjusted for balance; then the switch is thrown to 
the left, and the deflection due to $Q$ is read. A resistance shunted by a condenser is placed in the moving coil circuit of the series dynamometer so as to make the effective self-inductance of the circuit zero. This avoids a small correction which would otherwise enter. ${ }^{3}$ From the values of $R_{1}, R_{2}$ and $Q$ the ratio and phase angle may be readily determined. Unless the inductance of the shunts is negligible, corrections must be made to the phase angle.

Since the method was described a special adjustable noninductive shunt has been designed for use in the secondary. A series of bifilar manganin strips $T$ (Fig. 8), which are silver soldered to the lugs $D D$, are adjusted to the values $0.025,0.035,0.045$, etc., ohm. The lugs $D D$ have horizontal amalgamated surfaces which may be clamped by screws to corresponding amalgamated sur-

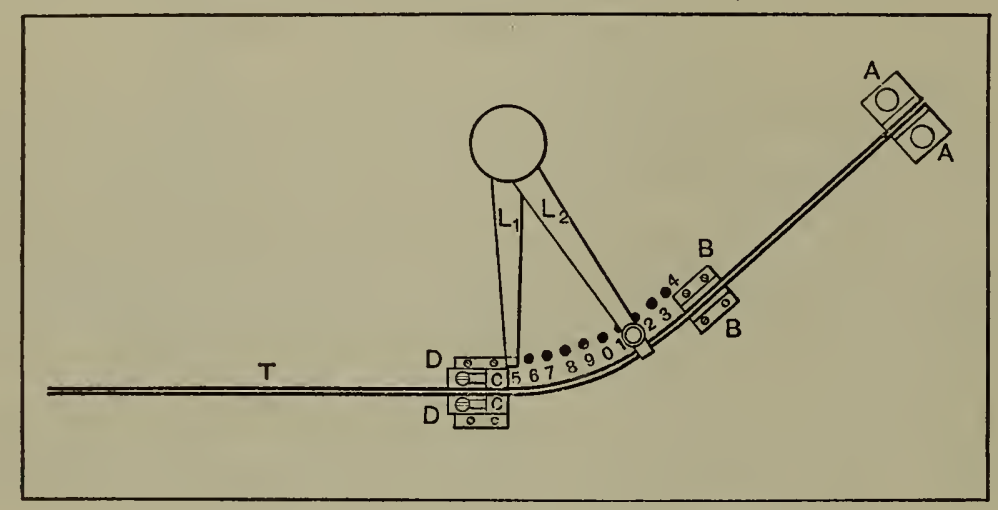

Fig. 8.-Adjustable noninductive resistance.

faces on the copper blocks $C C$. Between the blocks $B B$ and $C C$ are two strips of manganin separated by thin mica. The upper one has a resistance of o.or ohm, divided into steps of $0.00 \mathrm{I} \mathrm{ohm}$, and is connected to the numbered studs. The lower strip has a resistance of $0.00 \mathrm{I} \mathrm{ohm}$, divided into ro divisions, so that by estimating to tenths of a division the resistance may be read to 0.0000 I ohm. Copper strips connect the blocks $B B$ to the current terminals $A A$. The potential terminals are the levers $L_{1}$ and $L_{2}, L_{1}$ making contact with the studs, and the end of $L_{2}$ sliding on the edge of the thicker manganin strip, which is raised half a millimeter higher than the one to which the studs are connected. The capacity is ro amperes. At 60 cycles the phase angle is less than one minute.

\footnotetext{
${ }^{3}$ For a complete description of this method together with a derivation of the equations, sec Agnew and Iitch, this Bulletin, 6, p. 292, I909, Reprint I,30.
} 
It has been found that the error introduced by the variation in the resistance of the amalgamated contacts is entirely inappreciable for the accuracy required, which may be taken as one part in ten thousand.

Orlich ${ }^{4}$ has described a similar method in which a sensitive electrometer is used as the detector instead of the electrodynamometer.

Robinson ${ }^{5}$ has developed a rotating commutator with which he uses a direct current galvanometer as a detector in place of an electrometer or an electrodynamometer. This commutator is driven by a synchronous motor and rectifies the quadrature component $Q$ so that it may be measured by means of a direct-current galvanometer with all the requisite sensibility. The form factor enters into the reduction of the results.

This rotating commutator method has been further developed by Sharp and Crawford, ${ }^{6}$ who have also used a mutual inductance in the secondary to reduce the measurement of the quadrature component $Q$ to a null method. They have also suggested a method of using a mutual inductance instead of the shunts in primary and secondary circuits.

Laws ${ }^{7}$ has introduced a modification of the two-dynamometer arrangement in order to increase the sensibility at light load, which decreases with the square of the current. He substitutes for the series dynamometer one carrying a constant current which is kept in phase with the primary by an auxiliary device. This difficulty has been overcome in the work at the Bureau of Standards by the use of double-range instruments.

The point has been raised as to whether the two-dynamometer method gives the correct value of the ratio if the possibility of the introduction of distortion by the transformer be taken into account. For example, if we assume that the primary wave is sinusoidal and that the transformer introduces ro per cent of the third harmonic, then, since this harmonic component of the unbalanced emf $Q$ would give no torque in the dynamometer having a sinusoidal current in its field coil, the ratio of the effective values of the currents would differ from the ratio of the pri-

\footnotetext{
${ }^{4}$ Electrotechnische ZS., 30, p. 468 : 1909.

5 Trans. Amer. Inst. Elec. Eng., 28, p. 1005; 1909.

${ }^{6}$ Proc. Amer. Inst. Filec. Eng., 29, p. 1207, I910.

${ }^{7}$ Elec. World, 55, p. 223; I910.
} 
mary and secondary resistances by one-half the square of O.I, or 0.5 per cent. A similar statement would apply to the measured value of the phase angle.

But it is to be noticed that the rotating commutator method gives results which are open to the same theoretical objection, for the null setting gives the condition of equality of mean values rather than the equality of mean effective values. Fortunately the distortion introduced by the transformer is too small to be of any practical significance whatever. Yet it may be well to point out, even though it is of theoretical interest only, that the two-dynamometer method defines the proper ratio to be used in wattmeter measurements, that is the ratio of the primary to the undistorted part of the secondary. This follows from the principle that a harmonic present in the current coil of a wattmeter but not present in the emf wave adds nothing to the torque. This would of course not give the theoretically correct definition for an ammeter, but the accuracy required in alternating current measurements is not as great as that required in the measurement of power. Of course the same considerations hold for the electrometer method since they necessarily define the quantities in precisely the same way.

The phase angle does not suffer the same ambiguity in definition as does the ratio since it is used only in wattmeter or watthour meter measurements. Here the dynamometer and the electrometer methods give the theoretically correct definitions, not taking account of any harmonics that may be introduced by the transformer itself. While the rotating commutator method does include any harmonics introduced by the transformer, and while the percentage error thus introduced into the determination of the phase angle is much larger than in the case of the ratio, this error is still too small to be of practical significance, as will be shown later.

Another objection that has been urged against the rotating commutator method is that the form factor of the current wave used should theoretically be known, but here again the good wave forms given by modern alternators and the specification of practically a sinusoidal wave required in accurate measurements reduce the errors from this source to the limit of experimental error, and they need not therefore be considered. 


\section{CALCULATION OF RATIO AND PHASE ANGLE}

Fig. 9 is a vector diagram of the current transformer. If we let $\Phi=$ flux,

$I_{1}=$ primary current,

$I_{2}=$ secondary current,

$E_{2}=$ secondary electromotive force,

$n=$ ratio of turns of secondary to turns of primary,

$\phi=$ phase angle of secondary circuit,

$\theta=$ phase angle of transformer,

$M=$ magnetizing current,

$F=$ core loss component of exciting current,

$R=$ ratio of currents,

then we may consider that the flux $\Phi$ induces the emf. $E_{2}$ in the secondary giving rise to $I_{2}$ in the secondary and requiring a component in the primary opposite in phase and equal to $n I_{2}$ in magnitude.

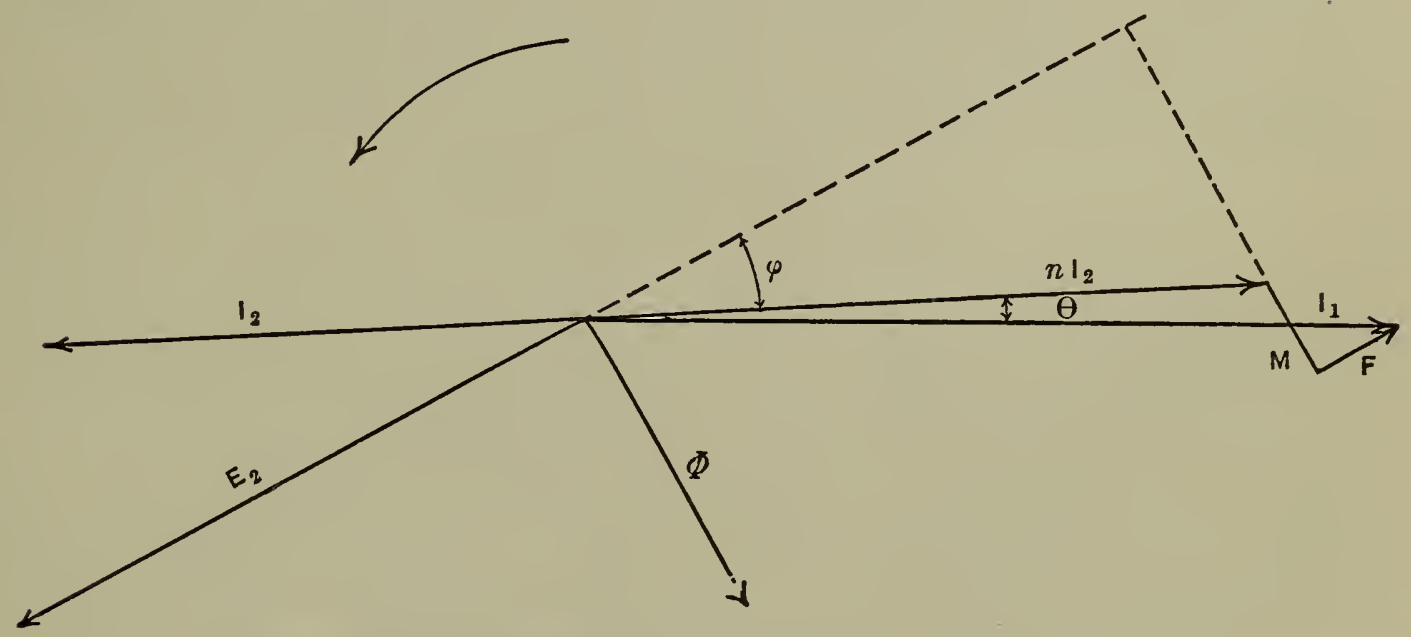

Fig. 9.-Vector diagram of current transformer on inductive load

But in order to maintain the flux the primary also has to furnish a magnetizing current $M$ in phase with the flux, and a core loss current $F$ opposite in phase to $E_{2}$. Hence, the total primary current is made up of $n I_{2}, M$, and $F$, taken in proper phase relations.

Projecting the vectors on the line of $n I_{2}$, we have

$$
I_{1} \cos \theta=n I_{2}+M \sin \varphi+F \cos \phi
$$

Projecting the vectors perpendicularly to the line of $n I_{2}$ gives

$$
I_{1} \sin \theta=M \cos \varphi-F \sin \varphi
$$


Squaring (I) and (2) and adding, neglecting the terms containing squares or products of $F$ and $M$,

$$
\begin{aligned}
I_{1}{ }^{2}=n^{2} & I_{2}{ }^{2}+2 n I_{2}(M \sin \varphi+\mathrm{F} \cos \varphi) \\
\therefore R=\frac{I_{1}}{I_{2}} & =\left[n^{2}+\frac{2 n}{I_{2}}(M \sin \varphi+F \cos \phi)\right]^{1 / 2} \\
& =n\left(\mathrm{I}+\frac{M \sin \varphi+F \cos \varphi}{n I_{2}}\right) \text { approx. } \\
& =n+\frac{M \sin \varphi+F \cos \varphi}{I_{2}}
\end{aligned}
$$

To determine the phase angle divide (2) by (r)

$$
\tan \theta=\frac{M \cos \phi-F \sin \phi}{n I_{2}+M \sin \phi+F \cos \phi}
$$

But as the exciting current is small in comparison to $n I_{2}$, we may write, for the purpose of computing the phase angle where an accuracy of only a few per cent is required

$$
\tan \theta=\frac{M \cos \varphi-F \sin \varphi}{n I_{2}}
$$

Formulas practically equivalent to (3) and (4) have been developed by Curtis, Drysdale, and Barbagelata. It has been found that the errors introduced by the approximations made in the derivation may safely be neglected, unless the measurements are carried to extremely low flux densities, but even in this case the uncertainties introduced by the magnetic history of the iron, etc., introduce uncertainties such as to make the use of the exact formula not worth while.

In case the load is noninductive,

$$
\sin \phi=\mathrm{O} \text { and } \cos \phi=\mathrm{I}
$$

and (3) and (4) reduce to the forms

$$
\begin{gathered}
R=n+\frac{F}{I_{2}} \\
\tan \theta=\frac{M}{n I_{2}}
\end{gathered}
$$


and the vector diagram takes the simple form shown in Fig. Io from which it is seen that the magnetizing current $M$ will determine the phase angle, but has very little effect upon the ratio,

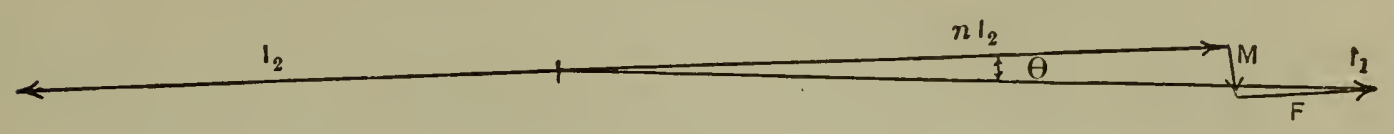

Fig. 10.-Vector diagram of current transformer on noninductive load.

while the core loss will determine the ratio, having practically no effect upon the phase angle.

The magnetizing and core loss components of the exciting current were measured directly by a method similar to that described

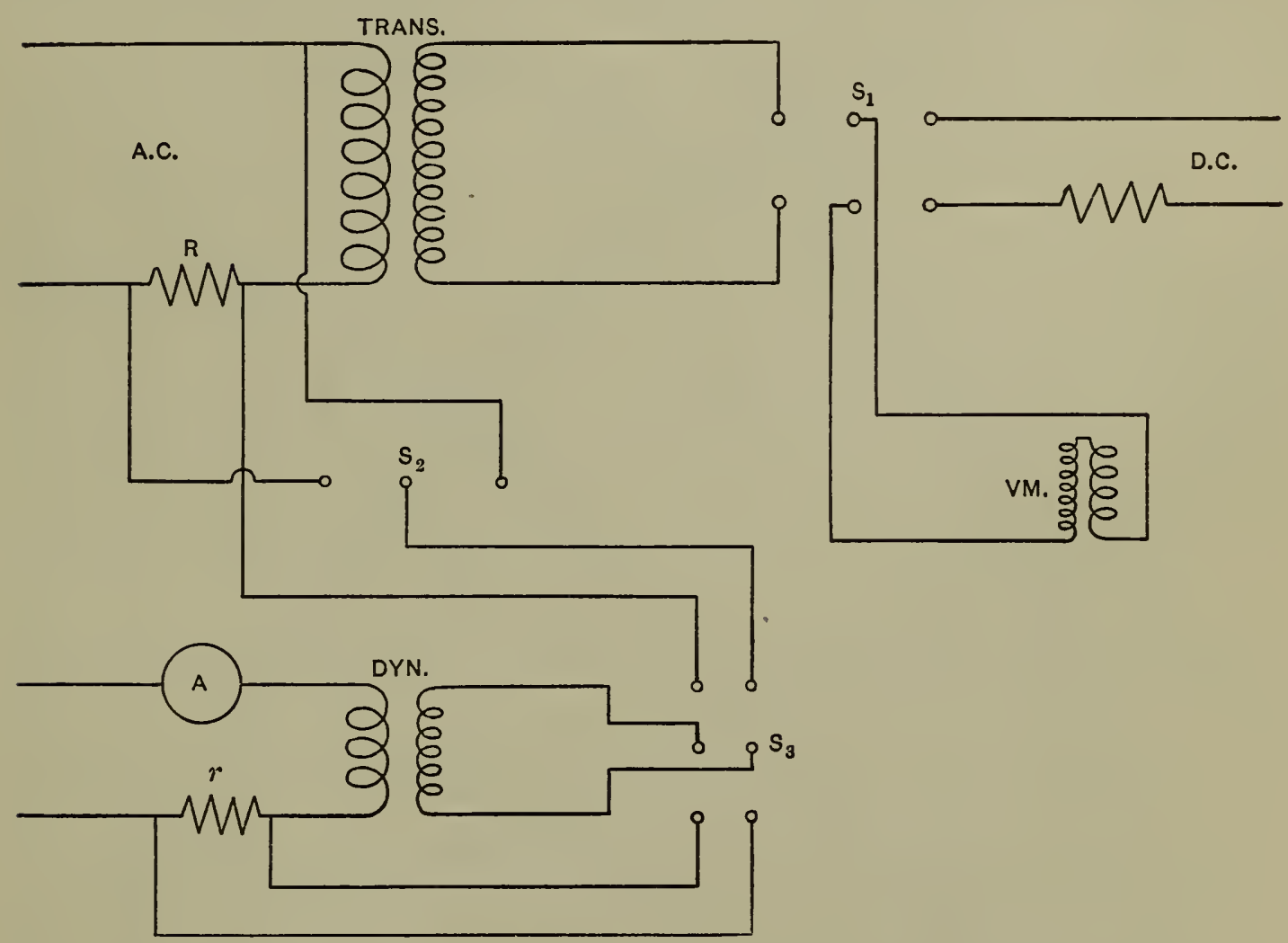

Fig. 11.-Connections for direct measurement of the magnetizing and core loss components of the exciting current.

by Sharp and Crawford ${ }^{8}$ and shown in Fig. I I. The secondary was adjusted by means of a sensitive reflecting voltmeter which could be calibrated on direct current by throwing the switch $S_{1}$.

${ }^{8}$ Proc. Amer. Inst. Elec. Eng., 29, p. r207; I9ro. 
The primary exciting current passed through a shunt $R$, the emf at the terminals of which could be applied to the moving coil of a

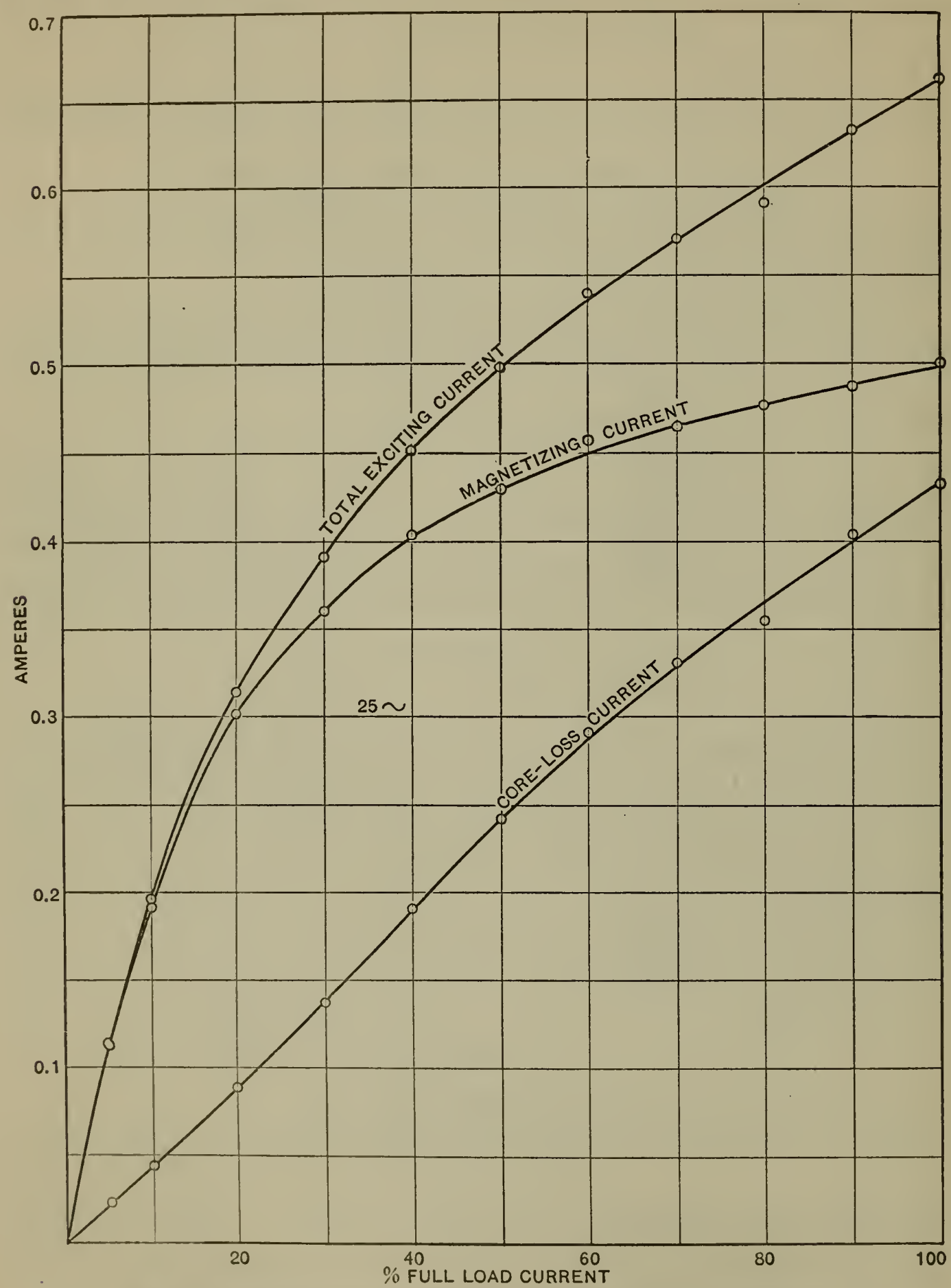

Fig. 12.-Components of exciting current at 25 cycles. Transformer $F$.

dynamometer by closing $S_{2}$ to the left, and $S_{3}$ up. The field of this dynamometer was excited from a phase shifting transformer. 
The procedure was to throw $S_{2}$ to the right, thus applying the primary voltage to the moving coil, and then to adjust the phase transformer until the dynamometer showed no deflection, which

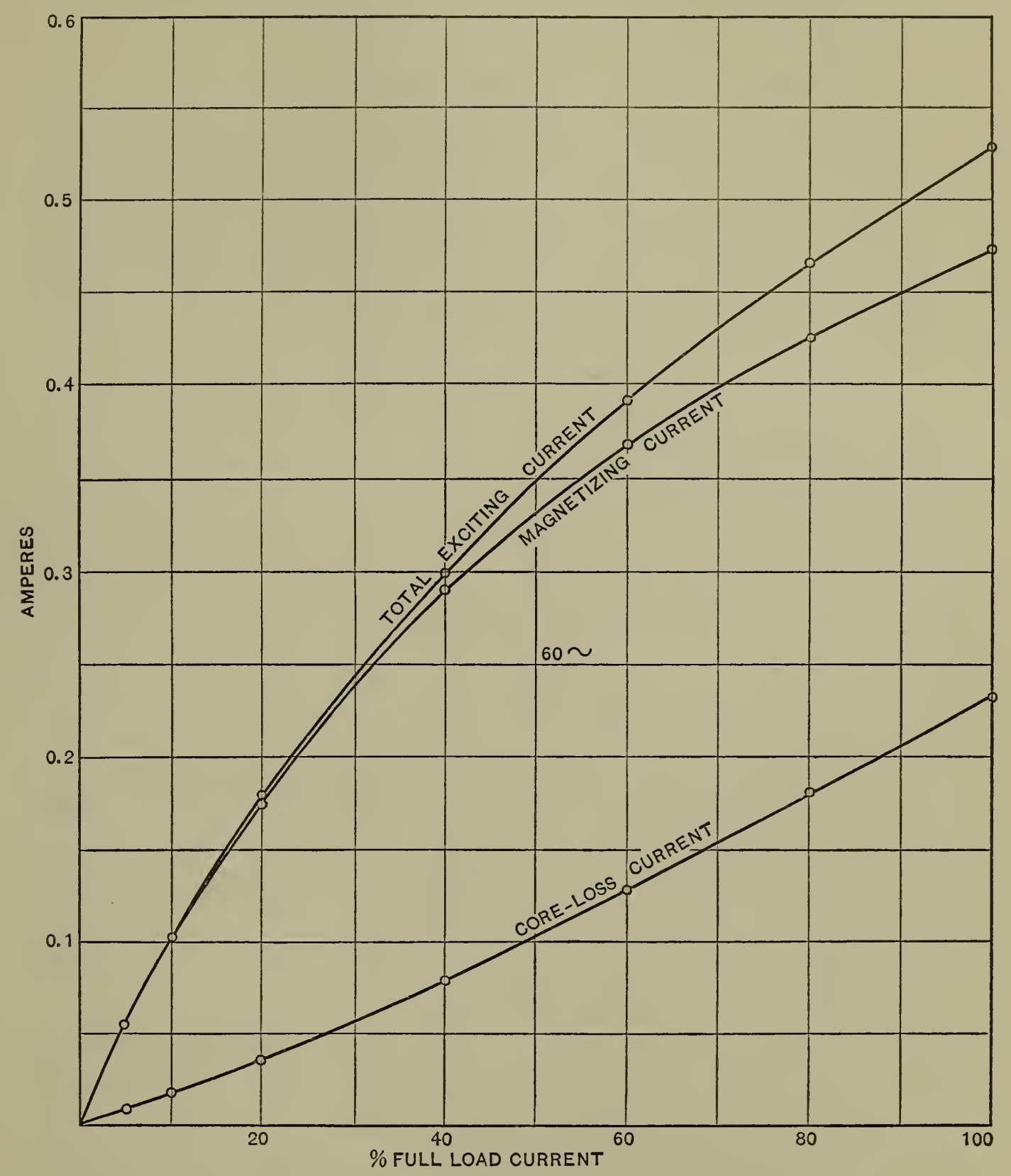

Fig. 13.-Components of exciting current at 60 cycles. Transformer $F$.

indicated that its field was in quadrature with the primary voltage of the transformer, and therefore in phase with the magnetizing current. $S_{2}$ was then thrown left and the magnetizing current 
read. The phase transformer was then turned through $90^{\circ}$ and the dynamometer again read, giving the core loss component. The dynamometer could be calibrated on alternating current by applying the voltage of a shunt $r$ to the moving coil of the dynamometer.

The core loss current, the magnetizing current and the total exciting current at 25 cycles for transformer $\mathrm{F}$ are shown in Fig. I 2 , and the same quantities for a frequency of 60 cycles in Fig. I 3 .

The ratio and phase angle were computed from this data and the results are indicated by the crosses in Figs. 4 and 5. The core losses at both 25 and 60 cycles are plotted in Fig. I4. The following are the constants of the transformer.

\section{CONSTANTS OF TRANSFORMER F}

Number primary turns 25 .

Number secondary turns 196.

Rated currents 40 and 5 amperes.

Secondary resistance $0.5 \mathrm{I} \mathrm{ohm}$.

Resistance of connected load $0.17 \mathrm{ohm}$.

Inductance of connected load $0.08 \mathrm{mh}$.

Maximum flux at 60 cycles, 290.

Maximum flux at 25 cycles, 700 .

It will be seen that the agreement between measured and computed values is very good, especially in the case of the 6o-cycle curve where the conditions are more favorable. The greatest discrepancy in the range from tenth to full load is but one part in three thousand. Of course, the possibility of the same error entering into both measurements is to be considered, as for example, errors due to wave distortion.

A check measurement of the ratio by an entirely different method was made, using the Northrup hot wire comparator. ${ }^{3}$ The makers of the instrument have devised a method for determining the ratio of current transformers, making use of a single potentiometer and a deflection instrument, for holding a direct current through one wire constant while the other wire was thrown from direct to alternating current. But as it was desired to get more sensitive readings than could be obtained by this method, 
an entirely different arrangement was adopted. The comparator was used as a transfer instrument by which the ratio of the two alternating currents was referred to the ratio of the readings of two potentiometers on direct current. The two hot wires of the instrument, which are indicated by the broken lines in Fig. I5, carry a concave mirror for observation with eyepiece and scale so that

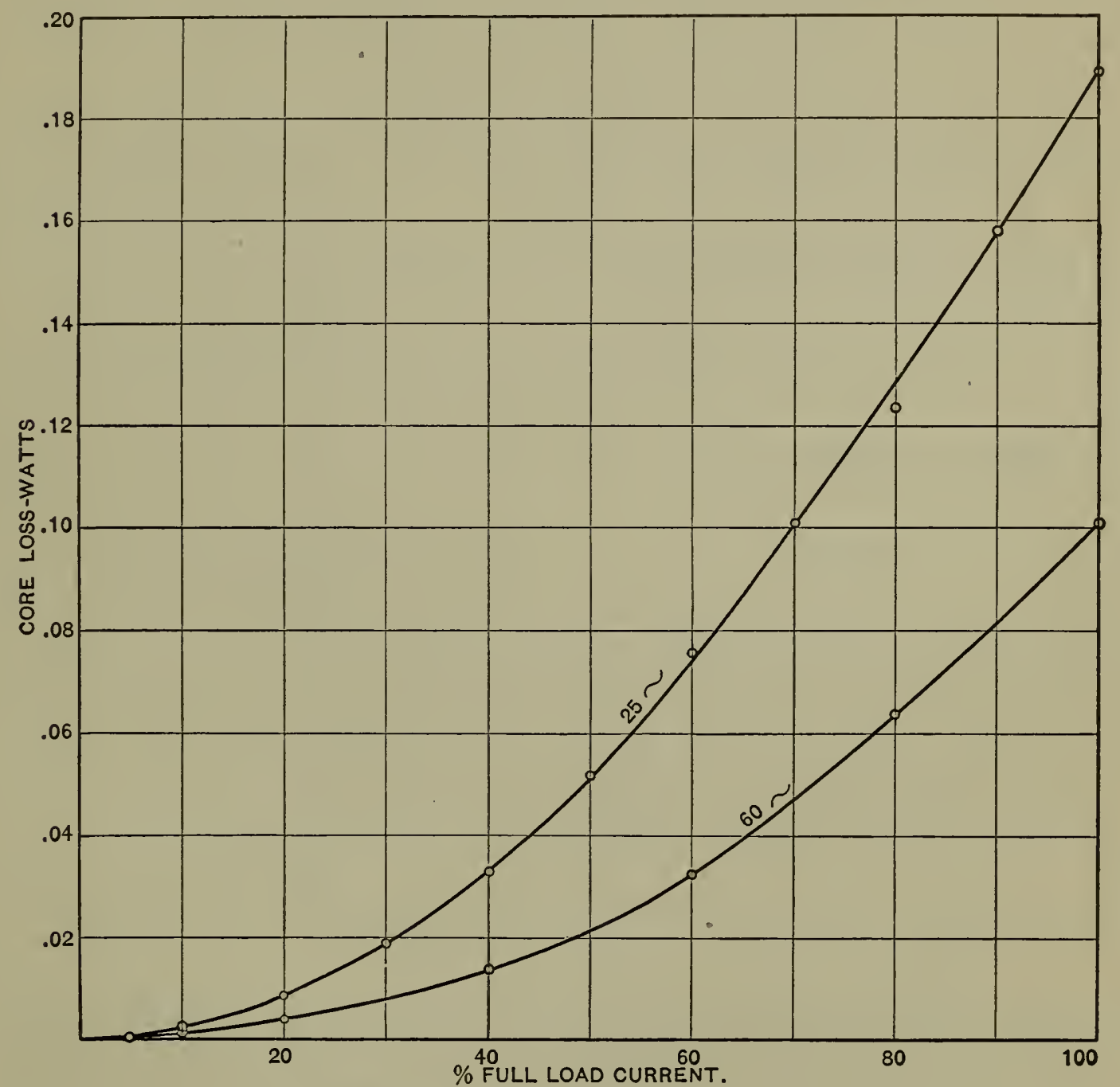

Fig. 14.-Core loss of Transformer $F$.

any difference in the expansion of the two wires gives a deflection of the scale. These were connected in parallel with two shunts $\mathrm{R}$ and $r$ which could be introduced either in the primary and secondary, respectively, or in two separate battery circuits adjusted to give the same current. The switches were arranged so that the comparator wires could be quickly and simultaneously 
thrown from alternating to direct current or vice versa. The direct currents were then adjusted so that the comparator showed no change when the switches were thrown in either direction. The direct currents must then be in the same ratio as the alternating currents; and they could be measured by the two potentiometers connected to the resistances $R_{1}$ and $R_{2}$. Two ammeters $A_{1}$ and $A_{2}$ were included in the circuits to make it possible to hold the direct current at the same value as the alternating,

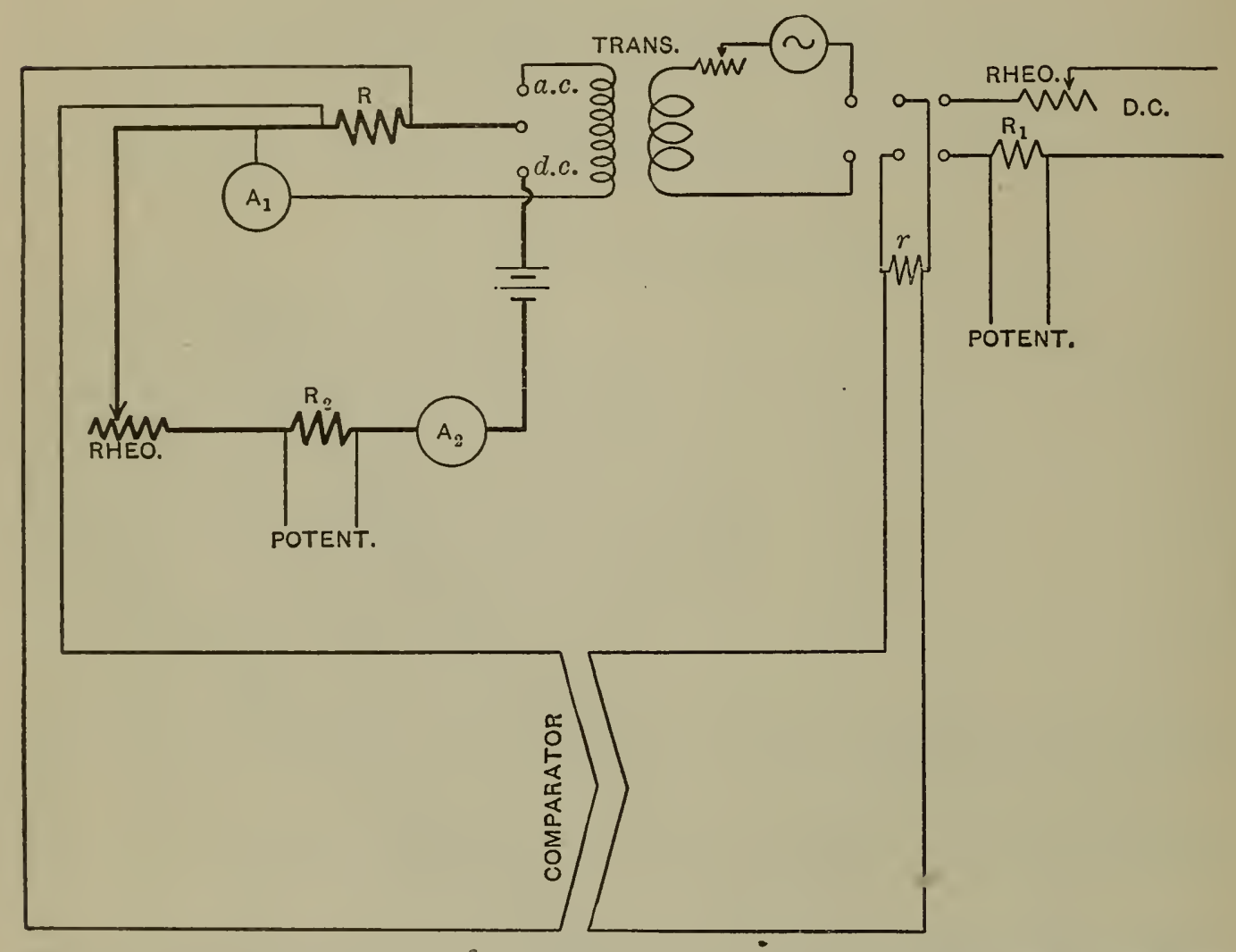

Fig. 15.-Arrangement for ratio determination by means of the Northrup hot wire comparator.

in order that the hot wires should be at the same temperature in both cases; but it is to be noted that the final ratio obtained does not depend upon the ammeter readings but upon those of the potentiometers. A further precaution was taken in adjusting a low resistance in the lead to one of the hot wires so that the two wires would expand and contract together when the current was thrown on or off.

The average of ro measurements gave the same ratio to the last figure (one part in eight thousand), at the full load current as was 
found by the two-dynamometer null method. (See Fig. 6.) Unfortunately this comparator method did not give great enough a sensibility to allow an adequate check of the values for the ratio at the lower current values. It is very difficult to see how the same error could enter into two such radically different methods of measurement, and as it will be shown that the distortion is negligible, it must be concluded that the forms of ratio curves found for transformer $\mathrm{F}$ are correct.

But so close an agreement between observed and computed values indicates that there must be some simple theoretical connection between the nature of the iron losses and the slope of the ratio curve.

\section{CONDITIONS FOR LINEAR RATIO AND PHASE ANGLE CURVES}

Such a relation may easily be shown to exist if we assume that the total iron losses may be expressed in the Steinmetz form

$$
W=K B^{c}
$$

where

$W=$ total iron loss in watts

$B=$ maximum flux density

$c, K=$ constants for a given transformer at a given frequency.

If $c$ be assumed to be rigorously constant, it can be shown that the ratio will increase with increasing secondary current when $c$ is greater than 2, and it will decrease with increasing current when $c$ is less than 2, provided the secondary circuit is noninductive. For by (5) the ratio

$$
R=n+\frac{F}{I_{2}}
$$

where $F$ is the core loss component of the exciting current and the term $\frac{F}{I_{2}}$ represents the departure from the ratio of turns. Both the current and the voltage may be taken as proportional to the maximum flux. If $E_{2}$ is the secondary voltage then since $F$ is measured in the primary and $E_{2}$ in the secondary we have

$$
F=\frac{n W}{E_{2}}
$$

$83226^{\circ}-11-9$ 
and

Hence from (7) and (8)

$$
\begin{aligned}
& I_{2}=B \times \text { Const. }=B K_{1} \\
& \therefore \frac{F}{I_{2}}=\frac{n W}{K_{1} B E}=\frac{n W}{K_{1} B K_{2} B} \\
& =\frac{n W}{K_{1} K_{2} B^{2}}
\end{aligned}
$$

$$
\begin{aligned}
\frac{F}{I_{2}} & =\frac{n K B^{c}}{K_{1} K_{2} B^{2}}=K_{3} B^{c-2} \\
\therefore R & =n+K_{3} B^{c-2} \\
\frac{d R}{d B} & =K_{3}(c-2) B^{c-3}
\end{aligned}
$$

But again, since $I_{2}$ is proportional to $B$,

$$
\frac{d R}{d I_{2}}=K_{4}(c-2) B^{c-3}
$$

This will be positive if $c$ is greater than 2 , and negative if it is less than 2. Hence, if the exponent $c$ for the total iron losses is equal to 2, the ratio curve becomes a horizontal straight line. If we assume the eddy-current loss to vary as $B^{2}$, then the same conclusions will apply to the hysteretic exponent alone. Formula (9) could readily be put in a form for quantitative determination of the ratio from the core loss by taking the actual value of $K_{4}$, but this is not necessary for our present purpose.

Some interesting relations connecting the shape of the ratio curve with the exponent, if the latter be treated as a constant, may be brought out by a development of equation (ro). Since the flux may be taken as proportional to the current this may be written

$$
\frac{d R}{d I_{2}}=K_{5}(c-2) I_{2}{ }^{c-3}
$$

To get the curvature we should, strictly, differentiate this with respect to the length of the curve, but since the curve is nearly horizontal we may, for approximate values, differentiate in respect to $I_{2}$ instead.

$$
\frac{d^{2} R}{d I_{2}{ }^{2}}=K_{5}(c-2)(c-3) I_{2}^{c-4}
$$


So that we have

$$
\begin{array}{ll}
\text { Ratio } & =n+K I_{2}^{c-2} \\
\text { Slope } & =K(c-2) I_{2}^{c-3} \\
\text { Curvature } & =K(c-2)(c-3) I_{2}{ }^{c-4}
\end{array}
$$

From the last of these equations it may be seen that the ratio curve will be a straight line (zero curvature) only when

$$
c=2 \text { or } c=3 \text {. }
$$

The matter is summarized in the following table.

\begin{tabular}{r|c|c|l}
\hline Exponent & Slope & Curvature & \multicolumn{1}{|c}{ Ratio Curve } \\
\cline { 2 - 3 } $\mathbf{c}<2$ & - & + & Slopes down; concave up \\
$\mathbf{c}=2$ & 0 & 0 & Horizontal straight line \\
$2<\mathbf{c}<3$ & + & - & Slopes up; concave down \\
$\mathbf{c}=3$ & + & 0 & Straight line, sloping up \\
$\mathbf{c}>3$ & + & + & Slopes up; concave up \\
\hline
\end{tabular}

The first case is the usual one, well illustrated by the typical curves of Fig. 2. While the straight line of the second case has never been completely realized, it is approached very nearly in some high-grade transformers with low impedance load, (compare the lower curve of Fig. 2). The third case of an upward sloping curve, concave downward is illustrated by the 6o-cycle curve for transformer $F$, (Fig. 4). Neither of the last two cases have ever been observed.

The simple theory as here outlined for a constant exponent is unable to account for a case in which the ratio passes through a maximum, as is the case with the 25-cycle curve for transformer $F$, (Fig. 4). The slope of this curve will be computed from the results of a more general treatment which will be given later.

It is seen from the factor $I_{2}{ }^{c-4}$, which enters the expression for the curvature, that the curvature will in general decrease with increasing current. The typical ratio curve (see Fig. 2) slopes down, is concave up, and the curvature decreases with increasing current, all of which are predicted by theory for the case of an exponent less than 2.

While the case of an assumed constant exponent thus agrees qualitatively with the experimental facts, it is quantitatively insufficient for precise measurements. Moreover, a more serious diffi- 
culty lies in the determination of the exponent when the latter is not a constant, and the variation is greater at the low flux densities used in the current transformer.

From the expressions for ratio and phase angle

$$
\begin{aligned}
& R=n+\frac{M \sin \varphi+F \cos \varphi}{I_{2}} \\
& \tan \theta=\frac{M \cos \varphi-F \sin \varphi}{n I_{2}}
\end{aligned}
$$

it may be seen that if the permeability were constant and the iron loss exponent were exactly 2 , then both the ratio and the phase angle curves would be horizontal straight lines. But this result would be expected since the transformer would then be equivalent to a circuit without iron, which should of course, give a constant ratio and phase angle.

\section{EXPONENTS AND THE RATIO OF VARIATION IN IRON LOSSES}

It has been assumed in what has gone before that the exponent $c$ is a true constant; otherwise, in differentiating (9) another term containing the derivative of $c$ with respect to $B$ would enter. It has long been known that an equation of the form

$$
W=K B^{c}
$$

will not accurately represent either the hysteresis loss or the total iron losses, and so it has become customary to speak of the exponent as varying slightly so as to force the equation to fit the experimental values. Numerous determinations have been published to show that the value of the exponent originally given by Steinmetz of $\mathrm{r} .6$ is only a sufficiently good mean for use over the limited range of inductions used in the design of power transformers, and that not only different kinds of iron give different values, but that for the same sample of iron the exponent varies with the induction.

Unfortunately, however, the methods that have been universally used to determine the exponent have depended upon the implicit assumption that the exponent is a true constant, and hence the values obtained are not actually exponents, but are, in most cases, the logarithmic derivative of $W$ with respect to $B$, and this becomes the exponent only in the case where it is a constant. In 
order to make the matter clear it will be well to consider first the methods that have been used to obtain the exponent from the experimental values.

The method most generally used at present is to plot $W$ against $B$ on logarithmic paper and measure the slope of the resulting curve at various values of $B$. Still considering $c$ a constant, if we differentiate the equation $W=K B^{c}$, first taking logarithms of both sides, .

$$
\begin{gathered}
\log W=\log K+c \log B \\
d(\log W)=c d(\log B) \\
c=\frac{d(\log W)}{d(\log B)}
\end{gathered}
$$

which shows that $c$ is the slope of the logarithmic curve. An equally accurate but less convenient method may be used with ordinary. cross section paper. Equation (I I) may be written in the form

$$
c=\frac{\frac{d W}{\frac{W}{d B}}}{B}=\frac{B}{W} \cdot \frac{d W}{d B}
$$

from which the value of $c$ at any point of the curve is seen to depend upon the values of $W, B$, and the slope of the curve at the point. These three quantities must be expressed in consistent units, as the slope $\frac{d W}{d B}$ is no longer a simple geometrical ratio as in equation (I I), but has the dimensions of $\frac{W}{B}$. Another method which is often used is to solve for $c$ from the values at two points of the curve. Thus if

and

$$
\left.\begin{array}{l}
\left.W_{1}=\begin{array}{lll}
K & B_{1} \\
W_{2} & =K & B_{2}
\end{array}\right\}
\end{array}\right\}
$$

then

$$
c=\frac{\log \frac{W_{1}}{W_{2}}}{\log \frac{B_{1}}{B_{2}}}=\frac{\log W_{1}-\log W_{2}}{\log B_{1}-\log B_{2}}
$$

Common logarithms may be used in either (I I) or (I4).

None of these methods is correct if we are dealing with a variable exponent for then in differentiating to get (I I) and (I 2) we should 
have had to take account of the variation of $c$ with respect to $B$, and evidently (14) could not have been deduced at all since we should have had different values of $c$ in (13). This will appear more clearly in an examination of the general case in which

$$
W=K B^{z}
$$

where the exponent $z$ is now a variable. It may first be said that suggestions have been made that $K$ should be considered to vary so as to fit the observations to some sort of a curve, and some writers have even treated both the coefficient and the exponent as variables, which is manifestly absurd. To consider that we have an exponent which varies slightly about a mean introduces complications, as will be shown, which greatly limits its usefulness, while any attempt to treat such a formula containing a variable coefficient can accomplish nothing since in its very simplest form the exponent would reduce to unity which merely brings us back to the measured values of $B$ and $W$.

To determine, then, the relations which follow by treating the coefficient as constant and the exponent as a variable, write (I5) in the logarithmic form,

Differentiating,

$$
\log W=\log K+z \log B
$$

$$
\begin{aligned}
& \frac{d W}{W}=\log B d z+z \frac{d B}{B} \\
& \frac{d W}{\frac{W}{B}}=z+B \log B \frac{d z}{d B}
\end{aligned}
$$

The left-hand member of this equation is the logarithmic derivative of $W$ with regard to $B$, or the slope of the curve obtained by plotting $W$ against $B$ on logarithmic coordinate paper. If $z$ is a constant the last term becomes zero, the curve becomes a straight line, and the slope of the logarithmic curve is the exponent. But if $z$ is not zero the value of the exponent from equation (I6) is

$$
=\frac{\frac{d W}{d B}}{\frac{d B}{B}}-B \log B \frac{d z}{d B}
$$


This shows that the logarithmic derivative is not the same as the exponent, as is tacitly assumed in the methods in common use in the determination of the exponent where the latter varies. The last term in (I 7 ) is entirely neglected in the methods which make use of either logarithmic or ordinary coordinate paper, as what is measured in these cases is merely the logarithmic derivative. Similarly the method of solving for the exponent by using values at two points of the curve will not give even the average value of the exponent over the range taken, as it is generally believed to do, for by equation ( 14 ) the quantity thus given is

$$
\frac{\log \frac{W_{1}}{W_{2}}}{\log \frac{B_{1}}{B}}
$$

Now, if we take $B_{2}$ very near $B_{1}$ we may replace $W_{1}, W_{2}, B_{1}, B_{2}$, by $W+\delta W, W, B+\delta B$, and $B$.

$$
\begin{gathered}
\frac{\log \frac{W_{1}}{W_{2}}}{\log \frac{B_{1}}{B_{2}}}=\frac{\log \frac{W+\delta W}{W}}{\log \frac{B+\delta B}{B}} \\
=\frac{\log \left(\mathrm{I}+\frac{\delta W}{W}\right)}{\log \left(\mathrm{I}+\frac{\delta B}{B}\right)}
\end{gathered}
$$

and we may replace $\log \left(\mathrm{I}+\frac{\delta W}{W}\right)$ by $\frac{\delta W}{W}$, which is the first term in its expansion, and similarly for $B$. This gives

$$
\frac{\frac{\delta W}{W}}{\frac{\delta B}{B}}
$$

which is the logarithmic derivative. Hence, this method gives a result which approaches that given by the other methods, namely, the slope of the logarithmic curve instead of the exponent. 
The slope of the logarithmic curve is, however, of much more practical importance than the true exponent, for the greatest use of such empirical relations is as interpolation formulas. And the logarithmic derivative is an exceedingly convenient form for interpolation, since for small variations it is the ratio of the percentage change in the dependent variable to the percentage change in the independent variable. The same confusion of it with a true exponent has developed in many similar experimental relations, such as, for example, the variation of the candlepower of lamps with voltage or with current or with the power supplied, and the variation of the resistance of fluids to objects moving through them. Such a confusion of terms is very unfortunate since it may lead to wrong conceptions. For example, in the case of incandescent lamps some of these logarithmic derivatives have numerical values very near the exponents in some of the theoretical radiation formulas, and it is misleading to express the relationship in exponents or powers of the dependent variable when the approximate agreement in numerical values may be entirely accidental.

It seems, therefore, advisable to have some simple expression which will of itself convey the meaning and be free from the objections that have prevented the common use of the term "logarithmic derivative", and I shall hereafter refer to this quantity as the "ratio of variation". For the suggestion of this term I am indebted to Mr. C. E. van Orstrand.

In order to bring out graphically the differences that may exist between the exponent and the ratio of variation in geometrical curves of the general parabolic form and whose exponents are of the order of magnitude of those met with in iron losses, in Fig. 16 the curve $y=x^{2+\frac{1}{x}}$ together with the exponent and the ratio of variation have been plotted. This curve was chosen as the values of the exponent and the ratio of variation are around 2 and both decrease with increasing values of $x$, for it is to be remembered that by equation (Io) the ratio curve of the current transformer has its slope determined by the exponent of the total iron losses, provided that exponent is constant. It will be shown later that the same statement will hold when the exponent 
is not constant if we use the ratio of variation instead of the exponent. In Fig. I 7 the curve $y=x^{1+0.1 x}$ together with the exponent and the ratio of variation are plotted. It will be seen that when the exponent is 2 the ratio of variation is 4.3. It happens that the curve for the ratio of variation for this purely mathematical curve is very much like one recently published for the hysteretic exponent for silicon steel at high inductions in which values as high as 3.6 were given, ${ }^{10}$ but as the method used in determining the "exponent" was to take the

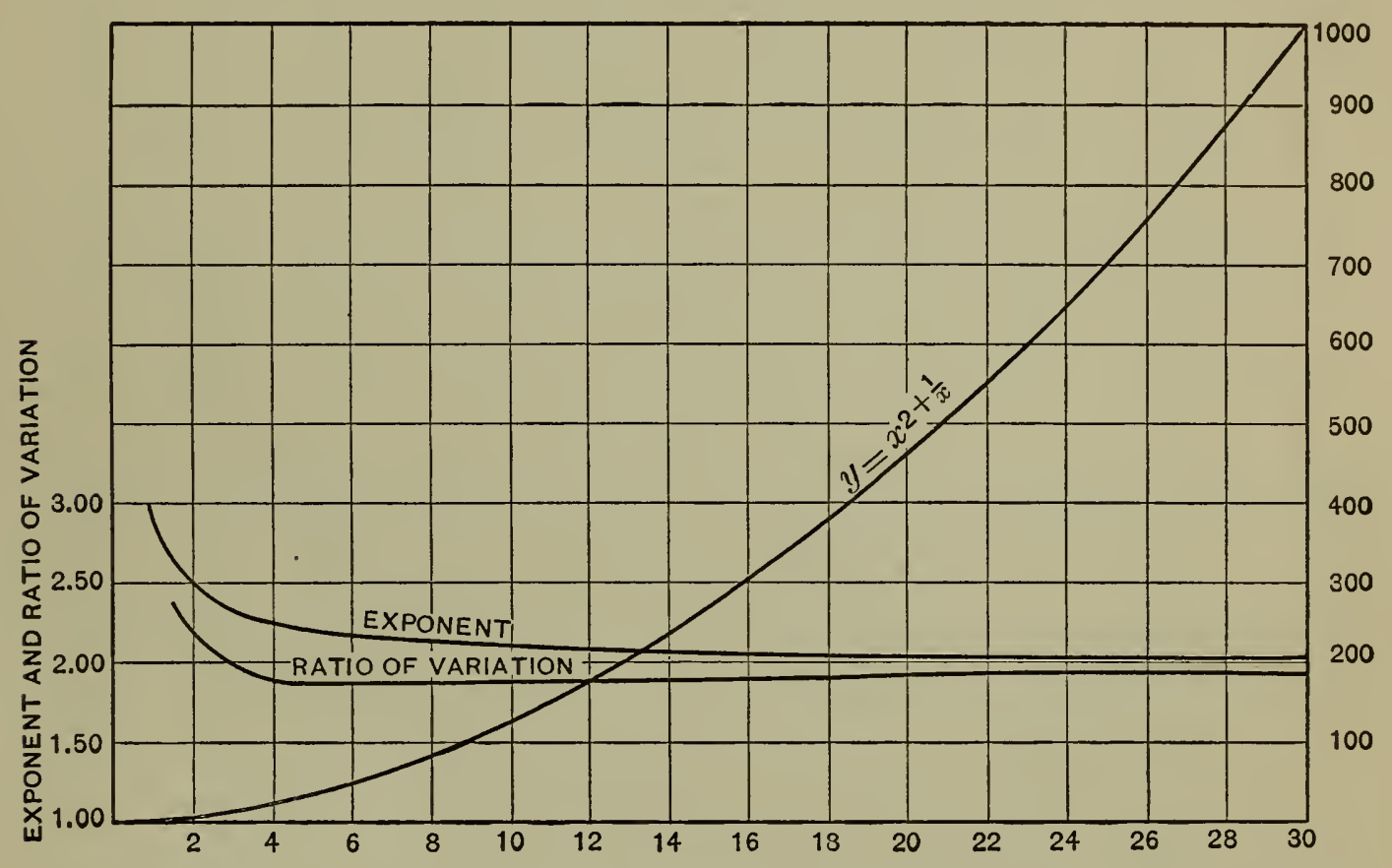

Fig. 16.-Showing the difference between the exponent and the ratio of variation in the curve $y=x^{2}+\frac{1}{x}$

slope of the loss curve plotted on logarithmic coordinate paper, evidently the quantity actually determined was the one here designated as the ratio of variation. Very probably the exponent as determined by methods to be explained presently would not have been greater than 2. The data given were insufficient to determine this point.

Since the last term in equation (I6) is positive it follows that the ratio of variation will be greater than the exponent when the latter

${ }^{0}$ W. J. Woolridge, Proc. Amer. Inst. Elec. Eng., 30, p. I39, I9I I. 
is increasing, and less when the exponent is decreasing. Consequently the exponent curve lies above in Fig. 16 and below in Fig. 17 .

If in the case of a curve whose exponent is changing slowly we choose two points of reference and solve for the exponent as if it were constant, one might expect to get a value somewhere near the mean of the actual values at the given points, but such is not the

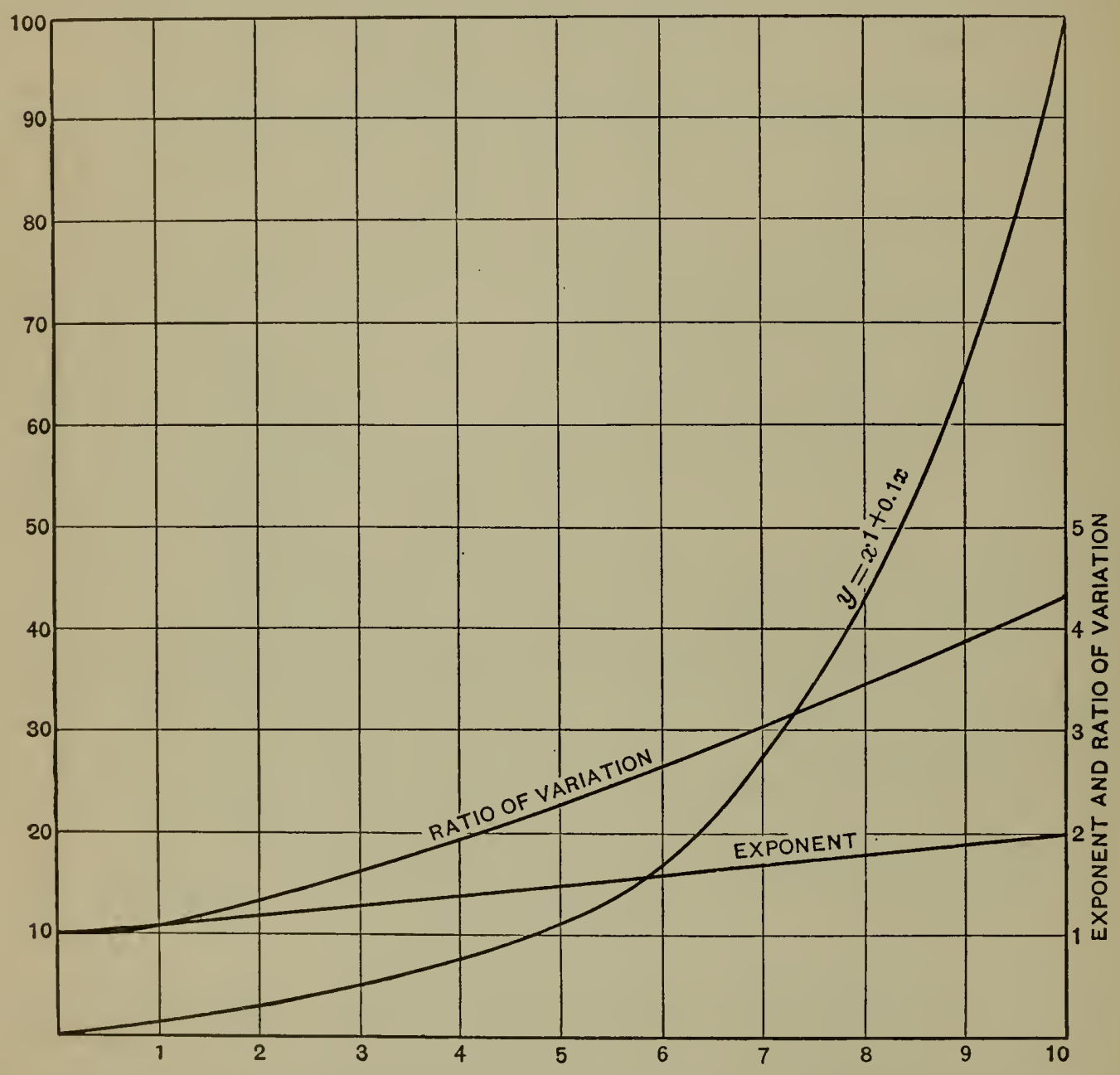

Fig. 17.-Showing the difference between the exponent and the ratio of variation in the curve $y=x^{1+0.1 x}$

case. It may be either greater or less than the actual value at either limit, according to circumstances. A more surprising result of the variation of the exponent is the effect that such a method has in making large changes in the coefficient. For example, if we consider the curve of Fig. 17 as an experimental curve and 
attempt to determine the coefficient and exponent by this method, which is the one that has been most frequently used in discussing changes in the Steinmetz exponent, we get the following results. The computed exponents are nearly identical with the ratio of variation:

\begin{tabular}{c|c|c}
\hline Limits used & Actual curve at midpoint of range & Computed equation \\
\hline$x=6$ to $x=8$ & $\begin{array}{l}y=x^{1 \cdot 7} \\
y=x^{1.8}\end{array}$ & $\begin{array}{l}y=0.075 x^{3.05} \\
y=0.0137 x^{3.86}\end{array}$ \\
\hline
\end{tabular}

The matter is not, however, so serious as these results indicate at first sight, for while the exponents have increased the coefficient has changed from unity to the small fractions, and either of the computed curves will give fair approximations throughout the small range for which it is computed. Yet it does emphasize the desirability of abandoning the use of the word "exponent" as applied to the results of such processes.

There are special cases in which the exponent may be determined with a fair degree of accuracy. If the ratio of variation is found to be constant over a range large enough to make sure of its constancy, it is in this region also the true exponent, and the coefficient may be calculated; and its value may be used in calculating the exponent at any other point of the curve. Also if the ratio of variation passes through either a maximum or a minimum its value at this point is the exponent, for a maximum or minimum in the ratio of variation means a point of inflection on the logarithmic curve, the condition for which is

$$
\begin{aligned}
\frac{d^{2}(\log y)}{d(\log x)^{2}} & =0 \\
\frac{d(\log y)}{d(\log x)} & =c \\
d(\log y) & =c d(\log x) \\
\log y & =c \log x+\log K \\
& =\log K x^{c} \\
y & =K x^{c}
\end{aligned}
$$


Now if we consider the exponent at the point $\left(x_{0} y_{0}\right)$ to be determined, then

From which

$$
\frac{y}{y_{0}}=\frac{K x^{2}}{K x_{0}^{z_{0}}}
$$

and

$$
z=\frac{\log y+Z_{\mathrm{o}} \log B_{\mathrm{o}}-\log y_{\mathrm{o}}}{\log B}
$$

By this method the exponent has been computed for the 25 -cycle core loss curve of transformer F (Fig. I8). The exponent is very

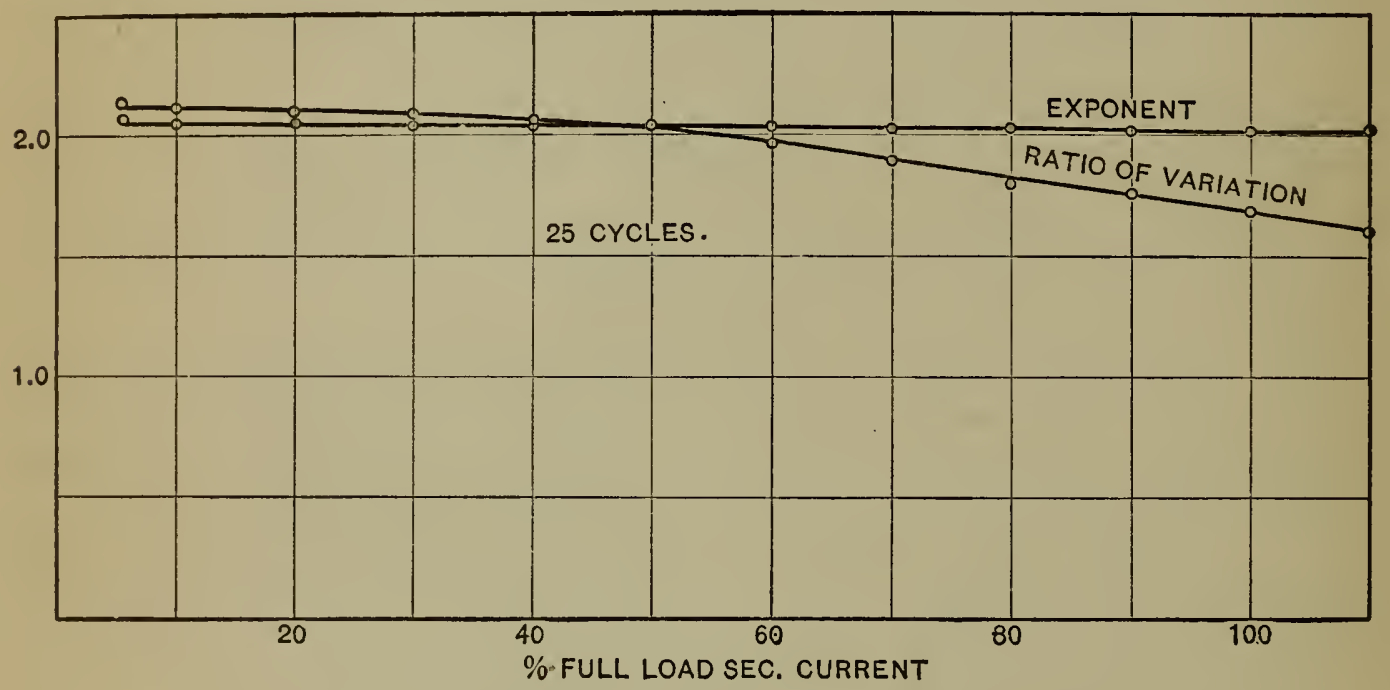

Fig. 18.-Exponent and the ratio of variation for transformer $F$.

much more nearly constant than the ratio of variation. In order to get an idea of the change of the exponent and of the ratio of variation over a wide range of flux densities the core loss data, determined by Mr. C. J. Huber on 2 special transformers, are platted on logarithmic paper in Fig. i g. The cores were of ring stampings of ordinary transformer steel, and the determinations were made by the wattmeter method at both 30 and 60 cycles. The core was first carefully demagnetized and the measurements made in the order of increasing flux.

The core of transformer $S_{1}$ was ordinary transformer steel, while that of $S_{3}$ was a silicon steel. The observed values of the total iron losses are given in Table $I$. The ratio of variation of the core loss of these transformers at 30 and at 60 cycles is plotted in 


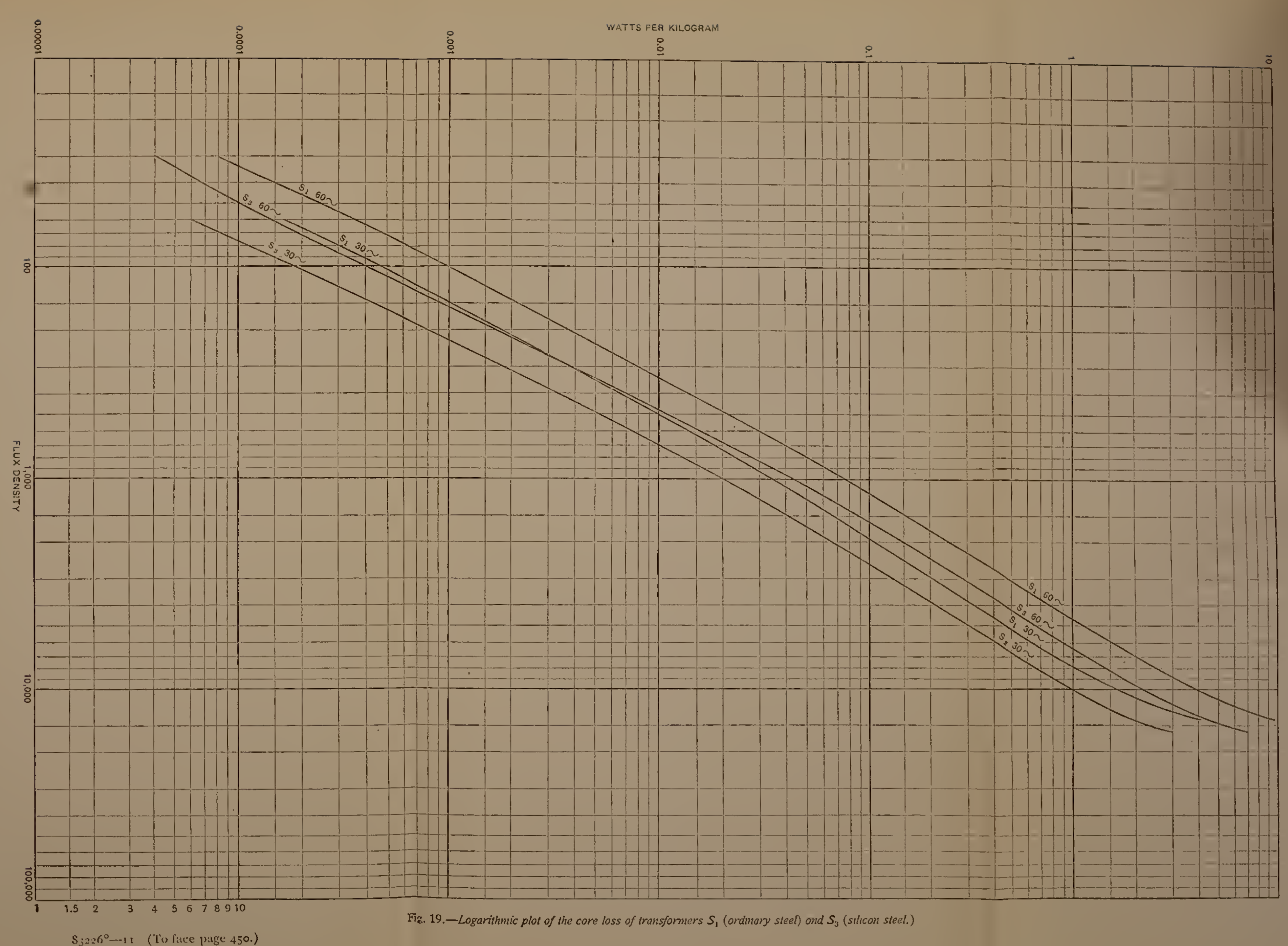


Figs. 20, 21, 22, and 23. Taking the ratio of variation as constant at 4000 lines, the exponents have been calculated by formula (I 8 )
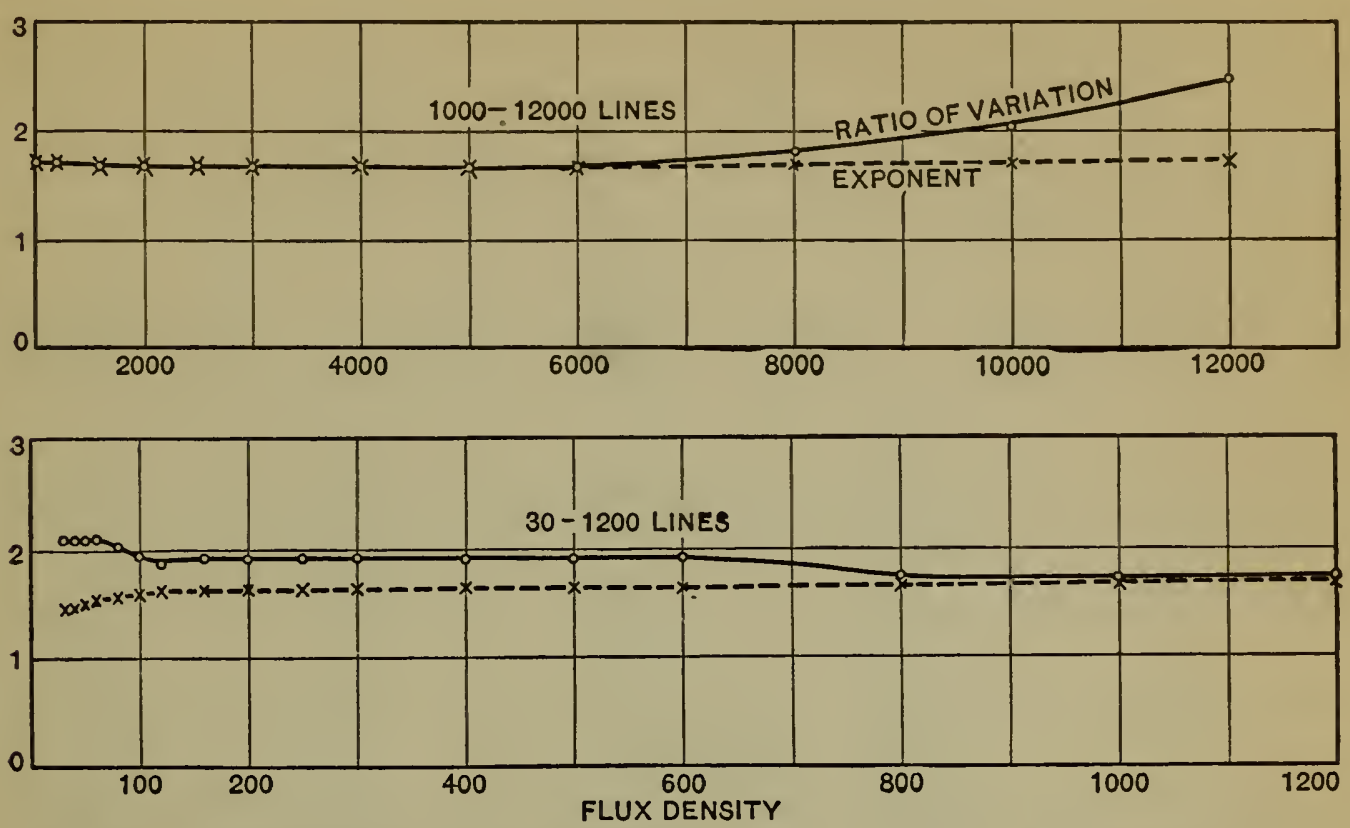

Fig. 20.-Exponent and ratio of variation for core loss of transformer $S_{1}$ (ordinary steel) 60 cycles.
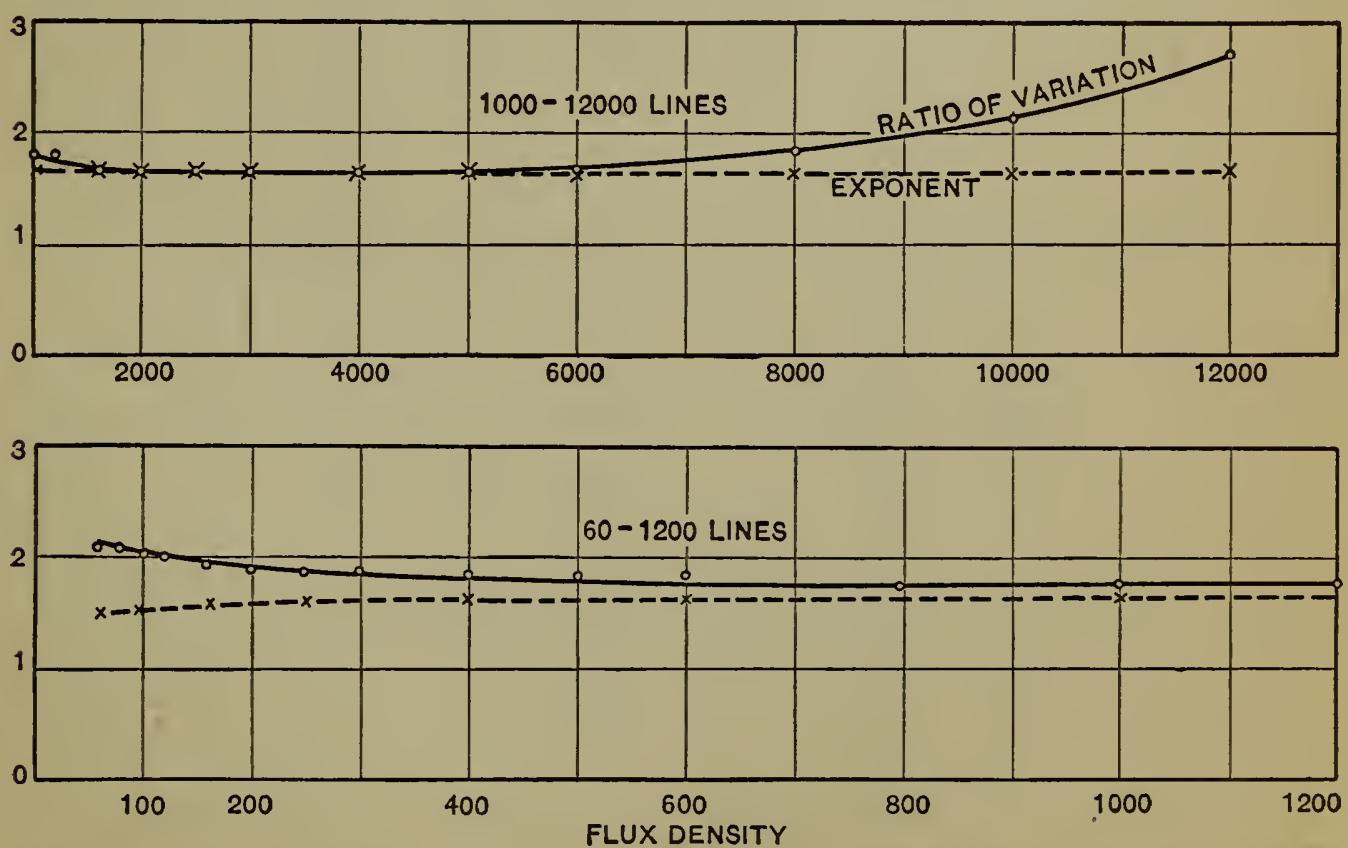

Fig. 21. Exponent and ratio of variation for core loss of transformer $S_{1}$ (ordinary steel) 30 cycles.

and the results plotted in the same figures. It is evident from the curves at high flux densities the ratio of variation reaches very 
high values for both the silicon and the ordinary steel, while the change in the exponent above 2000 lines is extremely slight. The
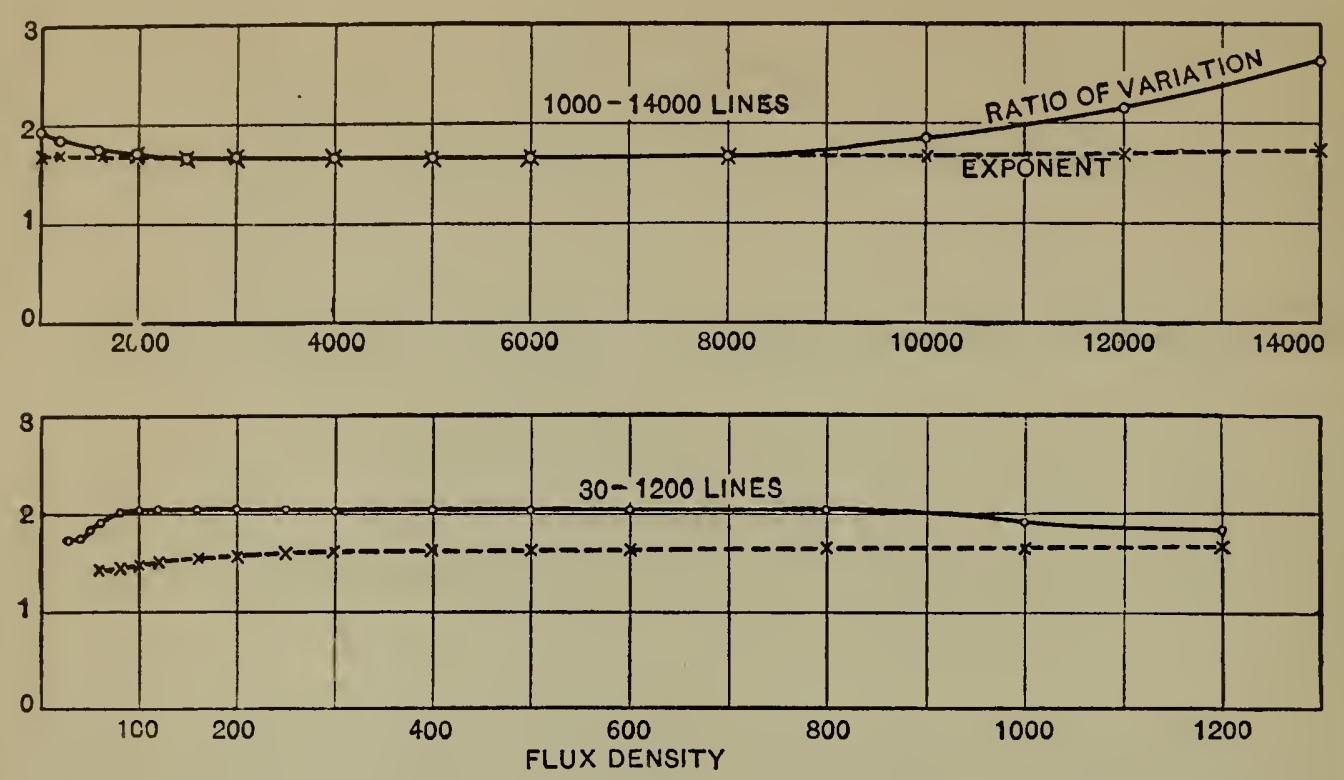

Fig. $22-$ Exponent and ratio of vanation for core loss of transformer $S_{3}$ (Silicon steel) 60 cycles.

exponent is more nearly constant than the ratio of variation, and after assuming a value at some given point may be determined
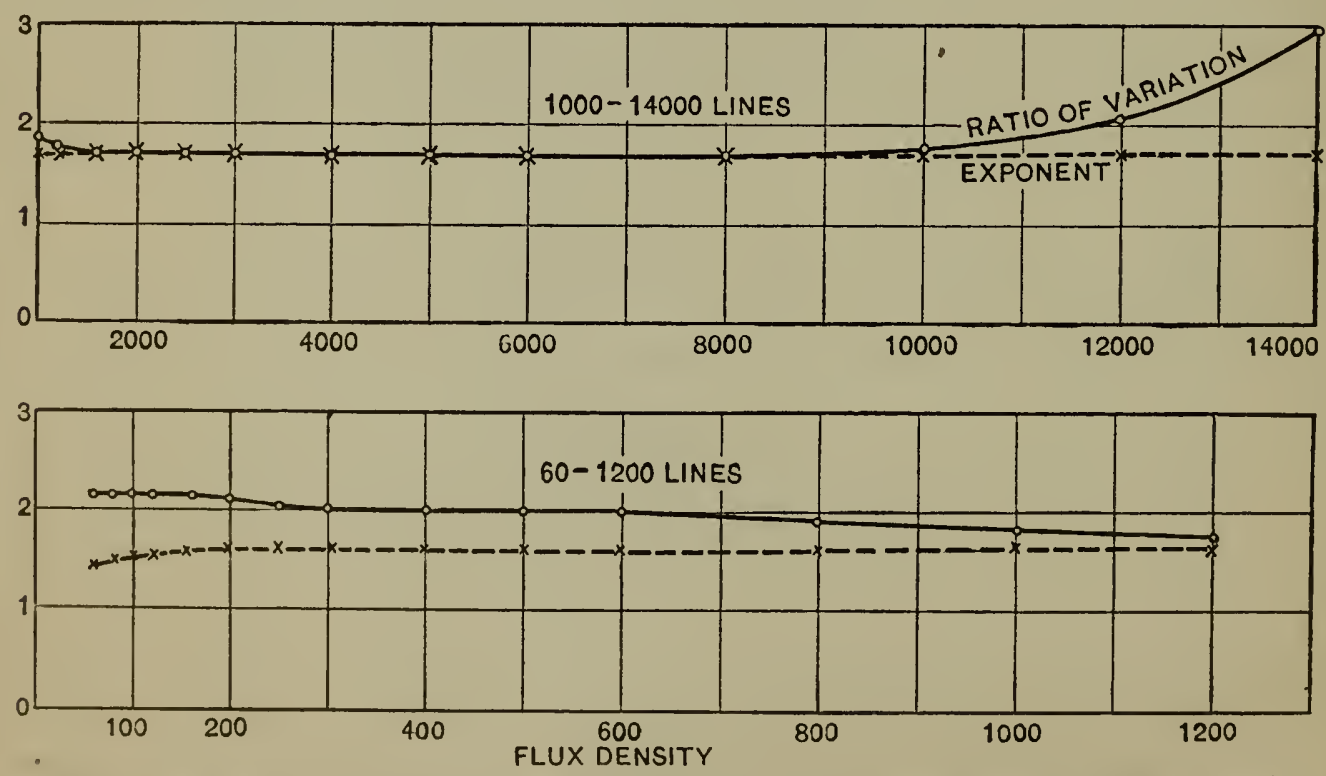

Fig. 23.-Exponent and ratio of variation for core loss of transformer $S_{3}$ (silicon steel) 30 cj'cles.

with a much greater relative accuracy. To bring this last fact out more clearly the computed values are given in Table II. 
TABLE I

Total iron losses (Watts per $\mathrm{Kg}$ )

\begin{tabular}{|c|c|c|c|c|}
\hline \multirow{2}{*}{ Flux } & \multicolumn{2}{|c|}{ Transformer $S_{1}$, ordinary steel } & \multicolumn{2}{|c|}{ Transformer $S_{3}$, silicon steel } \\
\hline & $30 \sim$ & $60 \sim$ & $30 \sim$ & $60 \sim$ \\
\hline 30 & & 0.000080 & & 0.000040 \\
\hline 40 & & .000145 & & .000050 \\
\hline 50 & & .000235 & & .000092 \\
\hline 60 & 0.000158 & .000353 & 0.00006 & .000135 \\
\hline 80 & .000294 & .000632 & .00011 & .000256 \\
\hline 100 & .000458 & .000971 & .00018 & .000390 \\
\hline 120 & .000666 & .00139 & .00027 & .000570 \\
\hline 160 & .00116 & .00242 & .00052 & .00103 \\
\hline 200 & .00179 & .00375 & .00079 & .00167 \\
\hline 250 & .00272 & .00585 & .00126 & .00270 \\
\hline 300 & .00385 & .00807 & .00185 & .00385 \\
\hline 400 & .00653 & .0140 & .00330 & .00694 \\
\hline 500 & .00985 & .0214 & .00518 & .0109 \\
\hline 600 & .0140 & .0298 & .00762 & .0154 \\
\hline 800 & .0233 & .0505 & .0131 & .0271 \\
\hline 1000 & .0344 & .0751 & .0199 & .0418 \\
\hline 1200 & .0468 & .104 & .0282 & .0590 \\
\hline 1600 & .0757 & .171 & .0465 & .0993 \\
\hline 2000 & .108 & .248 & .0680 & .147 \\
\hline 2500 & .154 & .358 & .0988 & .214 \\
\hline 3000 & .207 & .484 & .133 & .290 \\
\hline 4000 & .324 & .776 & .210 & .464 \\
\hline 5000 & .466 & 1.14 & .302 & .676 \\
\hline 6000 & .630 & 1.55 & .406 & .914 \\
\hline 8000 & 1.15 & 2.56 & .671 & 1.48 \\
\hline 10000 & 1.73 & 3.92 & .980 & 2.20 \\
\hline 12000 & 2.56 & 6.14 & 1.39 & 3.11 \\
\hline 14000 & 4.12 & 9.42 & 2.05 & 4.73 \\
\hline 16000 & 5.02 & & 3.01 & 6.83 \\
\hline
\end{tabular}


TABLE II

Exponents

\begin{tabular}{|c|c|c|c|c|}
\hline \multirow{2}{*}{ Flux density } & \multicolumn{2}{|c|}{ Silicon steel $\left(\mathrm{S}_{3}\right)$} & \multicolumn{2}{|c|}{ Ordinary steel $\left(\mathrm{S}_{1}\right)$} \\
\hline & $30 \sim$ & $60 \sim$ & $30 \sim$ & $60 \sim$ \\
\hline 30 & & 1.36 & & 1.49 \\
\hline 40 & & 1.32 & & 1.48 \\
\hline 50 & & 1.40 & & 1.51 \\
\hline 60 & 1.45 & 1.43 & 1.49 & 1.55 \\
\hline 80 & 1.50 & 1.49 & 1.49 & 1.58 \\
\hline 100 & 1.53 & 1.50 & 1.51 & 1.60 \\
\hline 120 & 1.55 & 1.53 & 1.53 & 1.61 \\
\hline 160 & 1.60 & 1.56 & 1.56 & 1.63 \\
\hline 200 & 1.61 & 1.58 & 1.57 & 1.64 \\
\hline 250 & 1.63 & 1.60 & 1.58 & 1.65 \\
\hline 300 & 1.64 & 1.62 & 1.59 & 1.66 \\
\hline 400 & 1.66 & 1.64 & 1.60 & 1.67 \\
\hline 500 & 1.67 & 1.65 & 1.61 & 1.68 \\
\hline 600 & 1.68 & 1.66 & 1.62 & 1.68 \\
\hline 800 & 1.70 & 1.67 & 1.63 & 1.69 \\
\hline 1000 & 1.70 & 1.68 & 1.63 & 1.69 \\
\hline 1200 & 1.70 & 1.68 & 1.63 & 1.69 \\
\hline 1600 & 1.71 & 1.69 & 1.64 & 1.70 \\
\hline 2000 & 1.71 & 1.70 & 1.63 & 1.69 \\
\hline 2500 & 1.70 & 1.69 & 1.63 & 1.69 \\
\hline 3000 & 1.70 & 1.69 & 1.63 & 1.69 \\
\hline 4000 & 1.70 & 1.69 & 1.64 & 1.69 \\
\hline 5000 & 1.70 & 1.69 & 1.63 & 1.69 \\
\hline 6000 & 1.70 & 1.69 & 1.63 & 1.69 \\
\hline 8000 & 1.70 & 1.69 & 1.65 & 1.70 \\
\hline 10000 & 1.70 & 1.69 & 1.65 & 1.70 \\
\hline 12000 & 1.70 & 1.69 & 1.66 & 1.71 \\
\hline 14000 & 1.71 & 1.71 & 1.68 & \\
\hline
\end{tabular}

The ratio of variation is, of course, independent of the units in which $W$ and $B$ are expressed, but the exponent is dependent upon the units used for $B$ although it does not depend upon the 
units in which $W$ is expressed. For, if instead of $B$ we write $\alpha B_{1}$, where $\alpha$ is a constant, the equation

$$
W=K B^{2}
$$

becomes

$$
\begin{aligned}
W & =K\left(\alpha B_{1}\right)^{z} \\
& =K \alpha^{z} B_{1}{ }^{2}
\end{aligned}
$$

In this form the coefficient contains the variable factor $\alpha^{z}$, which is not to be allowed, for the coefficient must be kept constant if such an equation is to have any useful meaning, as we have seen, and hence in order to keep the coefficient constant the exponent must take a new value. This may be seen more easily from equation ( 18 ) which is arranged in a form convenient for computing the exponent from experimental data. $B$ appears in the only term in the denominator while two of the terms of the numerator are independent of it, and hence to multiply $B$ by a constant will change the computed values of $z$. This difficulty disappears when the exponent is constant. It is sometimes a convenience to be able to change the unit in which $B$ is expressed, for example in shifting the decimal, or to use voltage or current instead of the flux, since they are usually proportional, and this is equivalent to a change of unit.

Another difficulty in computing the exponent from equation (I8) for values of $B$ approaching unity, is that small errors in the experimentally determined quantities introduce very large errors in the computed exponent. For points very near unity the equation fails entirely, as unity is an essential singular point of the function representing $z$, the denominator becoming zero. For all ordinary work this difficulty does not enter as only large values of $B$ are used. In Figs. 20 and $2 \mathrm{I}$ the lowest value of $B$ reached was 40. For the experimental determination at lower values recourse would have to be had to a null method such as that of Campbell ${ }^{11}$ who has used a mutual inductance and vibration galvanometer.

On the whole, the determination of the actual exponent is of little practical importance. It is much more difficult to deter-

${ }^{11}$ Proc. Phys. Soc. London, 22, p. 207; I910.

$8.3226^{\circ}-\mathrm{II} \longrightarrow \mathrm{IO}$ 
mine than the ratio of variation, it depends upon the unit in which the independent variable is expressed, and after it is once determined its use, as in interpolation, requires logarithms even for small intervals. The ratio of variation, that is, the slope of the logarithmic curve, is much more convenient to use in interpolating over small intervals. The fact that a constant exponent is the same as the ratio of variation has led to a failure to distinguish between them when the exponent is not constant, and this has introduced considerable confusion, not only in the literature of iron losses, but in the other fields in which the same ideas are made use of. Many attempts have been made to measure the variation of the exponent by methods which assume a constant exponent.

Recently Richter ${ }^{12}$ has proposed a two-constant formula to supplant the classical form, using only the first power and the square of $B$ so as to avoid fractional exponents. It is of the form

$$
W=a B+c B^{2}
$$

where $a$ and $c$ are constants. Jouaust ${ }^{13}$ has commented favorably on it. It is easily seen that the ratio of variation for iron whose losses follow such a law can not be greater than 2. For differentiating and dividing by the original equation

$$
\begin{gathered}
\frac{d W}{W}=\frac{a d B+2 c B d B}{a B+c B^{2}}=\frac{a+2 c B}{a+c B} \cdot \frac{d B}{B} \\
\frac{\frac{d W}{d B}}{B}=\frac{a+2 c B}{a+c B}
\end{gathered}
$$

and from its form the right member of this equation can not be less than $\mathrm{I}$, which it approaches for very small values of $B$, and it can not exceed 2, which it approaches for large values of $B$. It may also be seen from (20) that if we plot the ratio of variation against $B$ the resulting curve slopes upward throughout and hence has neither maxima nor minimum, or more rigorously, since the

\footnotetext{
${ }^{12}$ Electrotechnische ZS., Dec. 8, I9Io.

${ }^{13}$ Lumière Électrique, 13, p. 24I, I9II.
} 
left member is the ratio of variation we may get its slope by differentiating in regard to $B$.

$$
\frac{d}{d B}\left(\frac{\frac{d W}{W}}{\frac{d B}{B}}\right)=\frac{2 c(a+c B)-c(a+2 c B)}{(a+c B)^{2}}=\frac{a c}{(a+c B)^{2}}
$$

Which shows the slope to be always positive as stated.

Although Richter's formula may be very useful when applied to the relatively narrow limits of flux density used in commercial transformer design, it will evidently not meet the requirements over such wide ranges as shown for transformers $S_{1}$ and $S_{3}$ in Figs. 20 to 23 , or for such special cases as shown for transformer $F$

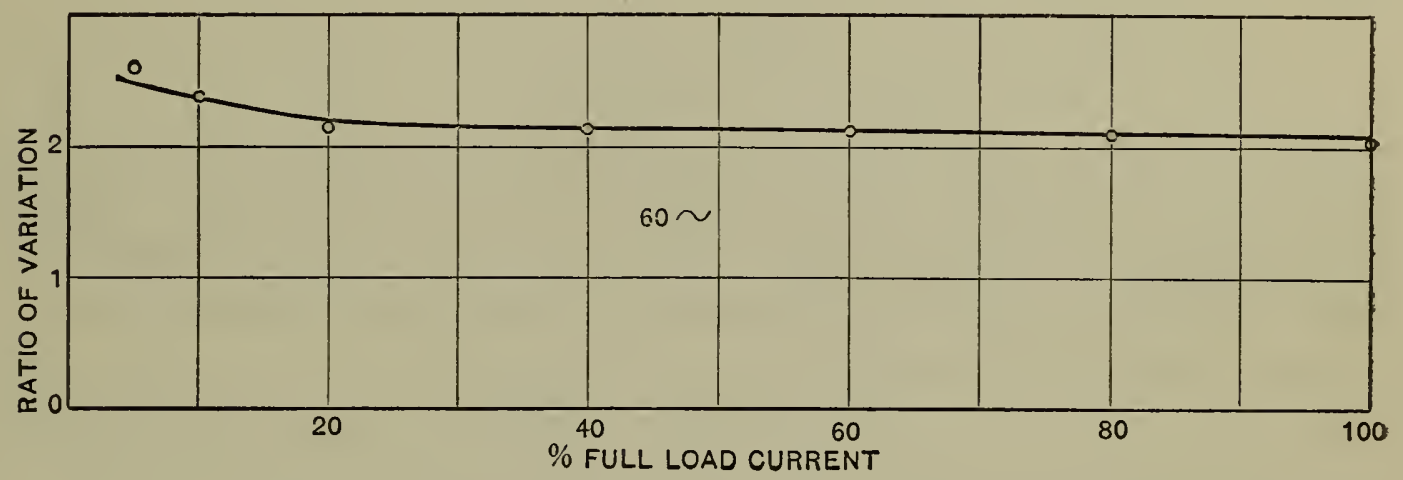

Fig. 24.-Ratio of variation for transformer $F$ at 60 cycles.

in Figs. I8 and 24, where the ratio of variation passes through maxima and minima, has negative slopes, values greater than 2, etc. Moreover, as our present interest is chiefly in exponents and ratios of variation attaining values of 2 or greater, the formula will not suffice.

\section{THE SLOPE OF THE RATIO CURVE DETERMINED BY THE RATIO OF VARIATION}

To return to the question of the ratio curve of the current transformer on noninductive load we had found, by means of equation (Io) that if the Steinmetz exponent for the total iron losses could be taken as constant then the ratio would increase with increasing current for exponents greater than 2, and would decrease with increasing current for exponents less than 2, the 
latter being the type of curve almost universally met with. We were unable to compare this theory with experimental results as experiment showed that the exponent was not constant. This led to the foregoing investigation of the relation between the exponent and the ratio of variation, and of the methods used in their determination, and we may now find a more general condition which determines the slope of the transformer ratio curve.

The previous statement of the dependence of the slope of the ratio curve upon the value of a constant exponent holds in exactly the same way for the general case of a variable exponent if the ratio of variation be substituted for the exponent. In fact the development may be made without assuming any empirical relation between the flux and the total loss. By equation (5) the ratio

$$
R=n+\frac{F}{I_{2}}
$$

where $n$ is the number of turns and $F$ the core loss component of the exciting current. If $W$ is the core loss, $E_{1}, E_{2}$ the primary and secondary voltages, and $r_{2}$ the secondary resistance then

$$
F=\frac{W}{E_{1}}=\frac{n W}{E_{2}}=\frac{n W}{r_{2} I_{2}}
$$

and the ratio becomes

Differentiating,

$$
R=n+\frac{n W}{r_{2} I_{2}^{2}}
$$

$$
\frac{d R}{d I}=\frac{n}{r_{2}} \cdot \frac{I_{2} \frac{d W}{d I_{2}}-2 W}{I_{2}{ }^{3}}=\frac{n W}{r_{2} I_{2}{ }^{3}}\left(\frac{\frac{d W}{W}}{\frac{d I_{2}}{I_{2}}}-2\right)
$$

Replacing $F$ from (2 I)

$$
\frac{d R}{d I}=\frac{F}{I_{2}{ }^{2}}\left(\frac{\frac{d W}{W}}{\frac{d I_{2}}{I_{2}}}-2\right)
$$

The fraction within the parentheses is the ratio of variation of the core loss with respect to the current, but since the current is 
proportional to the flux, this is the same as the ratio of variation with respect to the flux. Hence we may also write

$$
\frac{d R}{d I}=\frac{F}{I_{2}{ }^{2}}\left(\frac{\frac{d W}{W}}{\frac{d B}{B}}-2\right)
$$

Either (22) or (23) shows that the ratio of a current transformer will rise with increasing current when the ratio of variation is greater than 2, and that it decreases with increasing current when the ratio of variation is less than 2. This latter is, as already stated, almost universally the case.

That the experimental results obtained from the measurements of transformer $\mathrm{F}$ verify this conclusion may be seen from a comparison of the ratio curves of Fig. 4, with the curves of the ratio of variation of Figs. 18 and 24. At 60 cycles the ratio curve slopes upward throughout its length and in agreement with this the ratio of variation is greater than 2 over the whole range. In the curve at 25 cycles the ratio increases to half load and then slowly decreases to full load while in agreement with this the ratio of variation is greater than 2 for low currents and less than 2 for the larger currents, passing through the value 2 at approximately half load.

To test equation (22) quantitatively Table III has been computed for this transformer from the quantities plotted in the figures referred to.

TABLE III

Slope of Ratio Curve in Percentage Change of Ratio per Ampere

\begin{tabular}{l|r|r|r|r}
\hline \multirow{2}{*}{$\begin{array}{c}\text { Secondary } \\
\text { current }\end{array}$} & \multicolumn{2}{|c|}{60 cycles } & \multicolumn{2}{|c}{25 cycles } \\
\cline { 2 - 4 } & Calculated & Observed & Calculated & Observed \\
\hline 1 & +0.09 & $\begin{aligned}-0.09 \\
+.04\end{aligned}$ & $\begin{array}{l}+0.27 \\
+.04\end{array}$ & +0.09 \\
2 & +.06 & +.04 & .00 \\
2.5 & +.05 & +.03 & -.02 & -.02 \\
3 & +.02 & +.02 & -.07 & -.05 \\
4 & +.01 & +.01 & -.09 & -.07 \\
5 & & & & \\
\hline
\end{tabular}


With the exception of the I ampere point at 25 cycles the agreement is extremely satisfactory, the difference in the calculated and observed values of the ratio curve not exceeding 0.02 per cent for the ratio change per ampere. The discrepancy at the one point is due to the great difficulty in obtaining sufficiently accurate determinations of both the ratio and the core loss at the low loads. In fact the agreement at the $\mathrm{I}$ ampere 60 cycle point must be regarded as accidental. For currents less than an ampere the accuracy is not great enough to allow any comparison to be made, as is shown by plotted points in Fig. 4. This is not surprising when the magnitude of some of the quantities is considered. For example, at one ampere, 6o cycles, the core loss was but 0.003 watt and for many of the lower points the corrections for the energy taken by the measuring instruments was more than half the total quantity measured.

In Fig. 25 the loss and the ratio of variation for the core of a power transformer designated as transformer $\mathrm{G}$, are plotted for low flux densities, the maximum value reached being 235 gausses. The ratio of variation decreases from slightly over 2 at low voltage to slightly less than 2 at the highest voltage shown. When tested as a current transformer it gave a very flat curve as would be predicted from theory, and actually passed through a maximum; but the change in ratio was only a very few hundredths of a per cent.

A few other cases have been found in which the ratio of variation was slightly greater than 2 at low flux densities, as, for example, with transformer $S_{2}$, discussed in connection with Fig. I 9 , but no other case has been found in which the phenomenon of the ratio of transformation increasing with the current was anywhere nearly as pronounced as with transformer F. Reference has already been made to the fact that occasionally a transformer had been found whose ratio curve showed a tendency to turn down instead of up at the extreme low current end of the curves, and although passed over at the time as possibly due to errors of measurement, it seems entirely probable that these transformers would have shown core loss curves whose ratios of variation reached values of 2 or more. 


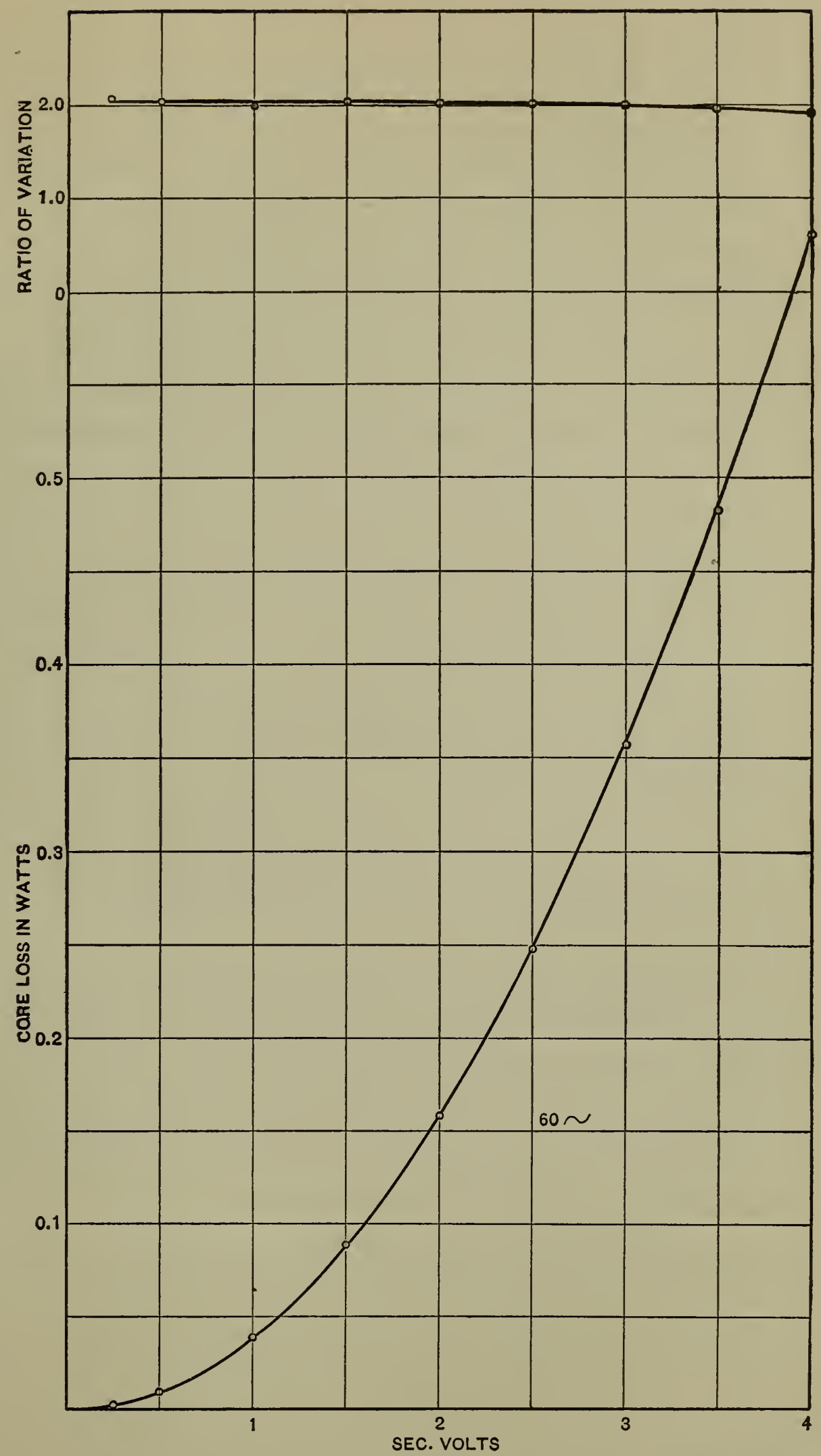

Fig. 25.-Core loss and ratio of variation for tranformer $G$ at 60 cycles. Maximum $B=235$. ! 
It might be supposed that all transformer iron might show high values of the ratio of variation if the measurements were pushed to low enough values of flux density. To determine this point recourse would have to be had to either very large masses of iron or to more sensitive methods, such as Campbell's, to which reference has already been made, for with some transformers the ratio of variation was well under 2 at the lowest flux densities that could be reached with the electrodynamometers used in these measurements. The large changes that magnetic history may introduce into the measured values of the permeability and the core loss at low densities where the effect is much greater is another disturbing factor in such measurements. It has been shown ${ }^{14}$ that considerable errors may be introduced into ratio and phase angle measurements by the variations in the magnetic constants due to the magnetic history of the core.

In the case of transformer $\mathrm{H}$ in which the ratio could be varied by series-parallel grouping of the primary windings the determination of the ratio and phase angle was carried to extremely low values of the current, and yet the ratio curve showed no tendency to turn down, (Fig. 26); on the contrary, the upward curvature steadily increases, indicating that the ratio of variation is still below 2. By special manipulation the sensibility did not fall below I/ 1500 even at 2.5 per cent of load. Incidentally these curves show how nearly the same form the ratio and phase angle curves may take. By changing the scale of one they would be nearly coincident.

Although there is need for a systematic study of iron losses at moderate and low flux densities, the data available being neither comprehensive nor systematic, at least nine other observers ${ }^{15}$ have reported values of the Steinmetz exponent as great as or greater than 2. As in nearly every case increasing or decreasing values of the "exponent" are reported, the values may be interpreted as the ratio of variation.

\footnotetext{
${ }^{14}$ For a detailed discussion, see Agnew and Fitch, this Bulletin, 6, p. 297, rgog. Reprint ${ }_{3}$ o.

${ }^{15}$ Raylcigh: Phil. Mag., 23, p. 225; r887. Ewing and Klassen: Phil. Trans. p. 985, 1893. Maurach: Ann. d. Phys., 311, p. 580; I901. Wild: Electrician, 56, 705; 1906. Sumpner: Electrician, 56, 768; rgo6. Wilson, Winston, and O'Dell: Proc. Roy. Soc., 80, p. 548; I908. Lloyd and Fisher: Bull. Bur. Standards, 5, p. 453; 1909. Reprint 109. Reed: Elec. Journal, 7, p. 36r; rgro. Wooldridge: Proc. Am. Inst. Elec. Eng., 30, p. I39, Jan. I9II.
} 
This dependence of the slope of the ratio curve upon the ratio of variation raises an interesting point in regard to methods sometimes used in the ratio determination. This method is to measure both primary and secondary currents separately by sensitive dynamometers, but as this requires the extra impedance of the dynamometer to be placed in the secondary circuit, the performance of the transformer is modified by this measuring instrument. Attempts have been made to correct for this by adding double the impedance so as to get a correction to apply in reducing the values to what would be obtained if the impedance of the instrument were not in circuit, or to reach the same end by determining the error thus introduced on a few transformers and thus to get a "blanket

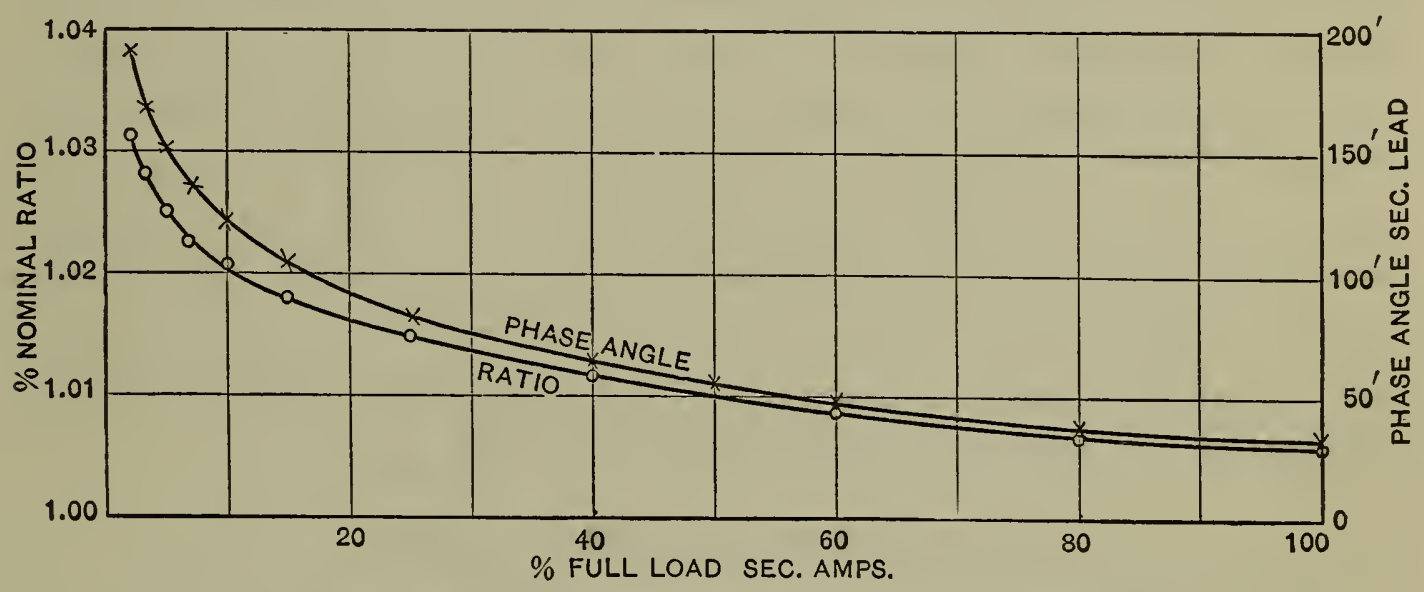

Fig. 26.-Ratio and phase angle curves of transformer $H$.

correction" to apply to all measurements. It is evident that this procedure may introduce considerable errors if the core should happen to have its ratio of variation pass through the value 2, and hence the ratio of transformation have a maximum. Accordingly, such a plan should not be used for the most accurate work, as it assumes precisely similar ratio of variation curves in all cases.

\section{BEARING ON DESIGN}

While the relation between the slope of the ratio curve and the ratio of variation has been established only for the case of noninductive load, it may be said that a transformer showing a good performance on noninductive load will usually give good ratio curves on inductive loads, and vice versa, so that while quantitatively the relation will not hold, it will do so in a general qualitative 
way. Evidently for a given total loss it would be better to select a grade of steel having a relatively high eddy current and low hysteresis loss, since the former would probably vary almost exactly as the square of the flux at the low densities used. Of course in this region the eddy current loss is usually less than the hysteresis loss, but experiments on different kinds of steel might result in finding one whose ratio of variation for the total iron losses would be nearly constant and have a value of approximately 2 . It would, of course, be desirable to have such a property coupled with a high permeability so as to keep the phase angle small.

It would also seem to follow that for a given frequency and a given secondary impedance load, the ratio performance might be better in a transformer using relatively thicker sheets, so as to increase the eddy currents, or even by the use of an auxiliary winding to be closed through a resistance. But for general work, where different frequencies and reactance loads are to be used it would not be advantageous, since the amount of the departure from the ratio of turns would be increased, and hence the change in the ratio resulting from a change in frequency or in impedance load would also be increased, although the curve would be flatter for any given frequency and impedance load.

\section{DISTORTION OF WAVE FORM}

There has been a considerable amount of discussion as to whether wave distortion in a current transformer can introduce appreciable errors in measurements in which the ratio or phase angle of the transformer enters. Of course in a circuit containing iron there must theoretically be some distortion, however small, and a greatly exaggerated importance has often been assigned to it. Robinson has shown by means of the oscillograph that even in the case of a complicated wave form the distortion can not be important, ${ }^{16}$ but no numerical determinations of the actual magnitude of the distortion have been published. A really satisfactory answer to such a question must depend upon quantitative data. Since, if distortion is allowed for, there are two possible definitions of the ratio of transformation and of the phase angle, and as there seemed to be a possibility of the same error entering into both the direct

16 Trans. Amer. Inst. Elec. Eng., 28, p. I005; 1909. 
determinations of the constants of the transformer and in the determination of the exciting current components, it was thought advisable to attempt some quantitative determinations of the magnitude of the distortion. The question of the effect of possible distortion on ratio and phase angle has already been discussed.

Two cases are to be distinguished, that of a sinusoidal primary current, and the much more complicated one in which the primary current is nonsinusoidal. In neither case, however, can the oscillograph or even the curve-tracer be made to give any but negative results, by merely analyzing the primary and secondary current waves separately. The amount of distortion is so minute that some indirect method must be employed.

The following method was used in the case of a sinusoidal primary current, taken from a star connected generator whose individual phases have very good waves. Two shunts $R_{1}$ and $R_{2}$

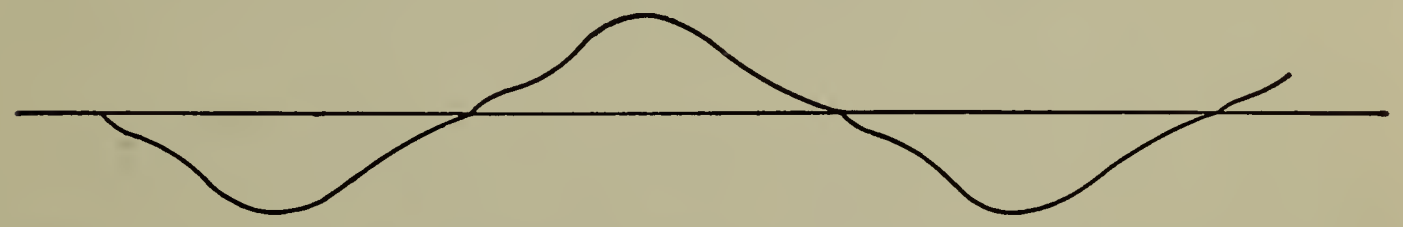

Fig. 27.-Wave form of the quadrature component in the secondary current of transformer $F$, as determined by the Rosa curve tracer. 25 cycles, full load.

were placed in the primary and secondary, respectively, and the value of $R_{2}$ adjusted to give the same in-phase drop as $I_{1} R_{1}$ as in the method of getting ratio. (See Fig. 6.) All the distortions present in $I_{2} R_{2}$ will appear in $Q$. Hence, if we in some way determine the amount of distortion in $Q$, it will immediately give us the distortion in the secondary current. The advantage of this procedure is that the harmonics present will form a very much larger part of $Q$ than of $I_{2} R_{2}$, and hence it is much like measuring a small difference directly rather than as the difference between the measurements of two large quantities. Small amounts of impurities in the primary wave will not appreciably affect the results.

In Fig. 27 is reproduced an example of the wave form of the complex emf $Q$, as determined by the Rosa curve-tracer. It was taken on transformer $\mathrm{F}$ at 25 cycles, 5 amperes secondary current. From an analysis of this wave by Thomson's method, the following data are computed for comparison with some of the data derived 
from the direct measurements of ratio, and of core loss and magnetizing current:

Effective value of 25 cycle component from curve........... 0.00496 volt

Effective value of 25 cycle component by dynamometer....... 0.00487 volt

Magnetizing current computed from curve................ 0.5 Ir amp

Magnetizing current observed ...................... $0.500 \mathrm{amp}$

Percentage of third harmonic in curve ............... r6.00 per cent

Percentage of fifth harmonic in curve.................. 3.00 per cent

Percentage of third harmonic in secondary current............ 0.13 per cent

Percentage of fifth harmonic in secondary current............ 0.03 per cent

Distortion due to third harmonic in terms of effective values..... . I part in I 000000

Distortion due to fifth harmonic in terms of effective values.... I I part in 20000000

The accuracy of the experimental data was not great enough to allow more than two harmonics to be computed, as the difficulties of tracing a wave of so small an emf are considerable. In fact, the close agreement shown must be in part accidental. In order to trace a wave the emf of which was only 5 millivolts, it was necessary to use a high sensibility galvanometer, to place extra wide contact pieces on the rotating contact maker, and to use various other precautions.

A direct analysis of the emf $Q$ was carried out by des Coudres' direct dynamometer method, ${ }^{17}$ in which the emf to be analyzed is applied to the moving coil of a dynamometer while a current from a machine giving any desired harmonic is passed through the fixed coil. The resulting deflection is due entirely to this particular harmonic. In order to obviate the difficulty of phase relations, the machines were run just out of synchronism, giving a continuous phase shift. The dynamometer would then deflect back and forth, following the continuously changing phase relation, the maximum travel either side of the zero giving a measure of the emf of the harmonic that was being determined. For the third harmonic the dynameter made three complete swings for one cycle of the synchronizing lamp, for the fifth, five, and so on. Only two harmonics could be determined in this way, on account of experimental difficulties, the chief of which was due to the inertia of the moving coil of the dynamometer, causing it to have an appreciable period of its own. For this reason the machines had to be run almost exactly in synchronism so that the changes in deflection would be slow. The deflections which it was possible to obtain were only a few millimeters.

${ }^{17}$ des Coudres, Electrotechnische ZS., 21 ; pp. $75^{2}$ and 770; 1900. 
The distortion found for a modern, high grade current transformer under various conditions of frequency, impedance load, and current is given in Table IV.

TABLE IV

Distortion Introduced by Transformer $J$. Primary Current Sinusoidal

\begin{tabular}{|c|c|c|c|c|}
\hline Frequency & Impedance in sec. & Sec. current & $\begin{array}{l}\text { Per cent third } \\
\text { harmonic }\end{array}$ & $\begin{array}{l}\text { Per cent fifth } \\
\text { harmonic }\end{array}$ \\
\hline 25 & 1 ammeter & 1 & 0.11 & 0.03 \\
\hline 25 & 1 ammeter & 2 & .08 & .03 \\
\hline 25 & I ammeter & 3 & .08 & .03 \\
\hline 25 & I ammeter & 4 & .08 & .03 \\
\hline 25 & 1 ammeter & 5 & .08 & .03 \\
\hline 25 & $1.6 \Omega$ & 1 & .31 & .09 \\
\hline 25 & $1.6 \sim$ & 2 & .33 & .06 \\
\hline 25 & $1.6 \Omega$ & 3 & .34 & .07 \\
\hline 25 & $1.6 \Omega$ & 4 & .34 & .07 \\
\hline 25 & $1.6 \curvearrowright$ & 5 & .36 & .08 \\
\hline 25 & $1 \bumpeq+8 \mathrm{mh}$ & 1 & .27 & .01 \\
\hline 25 & $1 \Omega+8 \mathrm{mh}$ & 2 & .26 & .01 \\
\hline 25 & $1 \Omega+8 \mathrm{mh}$ & 3 & .27 & .02 . \\
\hline 25 & $1 \sim+8 \mathrm{mh}$ & 4 & .28 & .03 \\
\hline 25 & $1 \sim+8 \mathrm{mh}$ & 5 & .28 & .05 \\
\hline 60 & 1 ammeter & 1 & .06 & .02 \\
\hline 60 & 1 ammeter & 2 & .05 & .03 \\
\hline 60 & 1 ammeter & 3 & .04 & .02 \\
\hline 60 & 1 ammeter & 4 & .04 & .02 \\
\hline 60 & 1 ammeter & 5 & .03 & .02 \\
\hline 60 & $1.6 \Omega$ & 1 & .15 & .06 \\
\hline 60 & $1.6 \Omega$ & 2 & .16 & .04 \\
\hline 60 & $1.6 \Omega$ & 3 & .16 & .04 \\
\hline 60 & $1.6 \Omega$ & 4 & .14 & .04 \\
\hline 60 & $1.6 \Omega$ & 5 & .14 & .04 \\
\hline 60 & $1 \sim+8 \mathrm{mh}$ & 1 & .19 & .03 \\
\hline 60 & $1 \Omega+8 \mathrm{mh}$ & 2 & .20 & .03 \\
\hline 60 & $1 \Omega+8 \mathrm{mh}$ & 3 & .22 & .03 \\
\hline 60 & $1 \Omega+8 \mathrm{mh}$ & 4 & .22 & .04 \\
\hline 60 & $1 \sim+8 \mathrm{mh}$ & 5 & .22 & .04 \\
\hline
\end{tabular}


The percentage of harmonic present in each case is seen to be independent of the current, so far as the accuracy of the method is concerned. Evidently the amount of distortion in a current transformer is extremely small, for a sinusoidal primary wave the highest figure obtained, and that under severe conditions, being but 0.36 per cent of the fundamental, and this adds less than one part in one hundred thousand to the effective value. About the same value is obtained for the 25 cycle, low impedance load as was obtained under similar conditions for transformer $F$, which was the transformer showing the peculiar ratio curves. In no case is the amount of fifth harmonic introduced great enough to change the ratio of effective values by as much as one part in a million.

In order to determine the distortion for the case of a nonsinusoidal primary wave the method was considerably modified. For the experiment two transformers having 5 to 5 ampere windings were chosen in order that precisely similar shunts might be used in primary and secondary, thus eliminating errors due to difference in the inductances of the shunts. The most essential modification of the method consisted in reducing the measured value of the quadrature resultant electromotive force $Q$ (Fig. 6) to zero, by introducing a variable self. inductance in the secondary so as to vary the phase angle of the transformer, in fact to bring it to zero. Under this condition if there were no distortion introduced by the transformer there would be no resultant emf whatever to be applied to the moving coil of the dynamometer, and hence any resultant that is found in $Q$ is due to distortion within the transformer. The diagram of connections is shown in Fig. 28. For convenience a dynamometer having 2 separate field coils for $\mathrm{I}$ and 5 amperes was used but two separate instruments would have done as well. The self-inductance of the moving coil was compensated and the coil set at the position of zero mutual inductance. Two motor-driven 2-phase alternators rigid on the same shaft and designed for 25 and 75 cycles were used as sources. The 5-ampere coil of the dynamometer could be thrown in or out of the primary circuit by the switch $M$, while the I-ampere coil could be connected to either phase of the 75 -cycle machine. 
The procedure was to throw $M$ to the right, $N$ up, and adjust $R_{2}$ for the ratio, $S$ being open. Then throw $M$ left, $N$ down, and adjust the self-inductance $L$ for no deflection or $Q$. To get both adjustments it was necessary to successively approximate, and it was found convenient also to change the frequency. The primary current was maintained constant at full load by the ammeter $A_{1}$. When these adjustments were made, with $M$ left, $N$ up, $S$ was thrown first left and the in-phase component of the distorted emf read on the upper dynamometer. $S$ was then thrown right and the quadrature component measured. $S$ con-

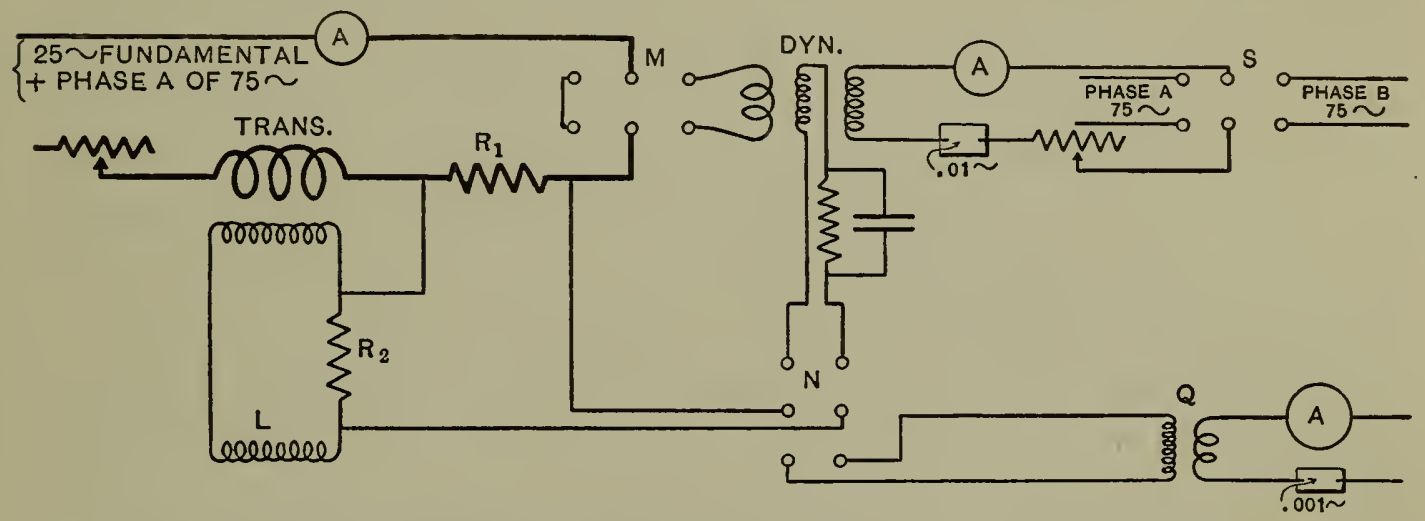

Fig. 28.-Connections for measuring the distortion in a current transformer with a nonsinusinusoidal primary wave.

nects the moving coil to the source of the third harmonic. The results which were obtained at a frequency of 38 cycles, are shown in Table V.

\section{TABLE V}

Distortion emf (volts)

\begin{tabular}{c|l|r|r|r|r}
\hline Transformer & Wave form & In phase & In quadrature & Total & \multicolumn{1}{c}{$\begin{array}{c}\text { Change in effective } \\
\text { value }\end{array}$} \\
\cline { 2 - 6 } $\mathrm{K}$ & $20 \%$ dimple & 0.00050 & 0.00017 & 0.00053 & 1 in 4000 \\
"، & $20 \%$ peak & 21 & 51 & 55 & 1 in 10000 \\
$\mathrm{~S}_{1}$ & $20 \%$ dimple & 77 & 28 & 82 & 1 in 2500 \\
$،$ & $20 \%$ peak & 32 & 33 & 46 & 1 in 6000 \\
\hline
\end{tabular}

In computing the change in the effective value only the in-phase component of the distortion emf was taken into account as the 
effect of the quadrature component would be entirely inappreciable. The current was 5 amperes, and $R_{1}$ and $R_{2}$ approximately $0.075 \mathrm{ohm}$.

Transformer $K$ was of an old type showing a poor ratio curve, and $S_{1}$ was a special transformer.

We may conclude from the results of these determinations of the magnitudes of the distortion introduced by current transformers, that the distortion is so small that it is of theoretical interest only, amounting as it does to less than one part in one hundred thousand in effective value when the primary current is sinusoidal, and to but one part in two thousand five hundred in the most unfavorable case, and that with 20 per cent of third harmonic in the primary wave. When we consider the good wave forms given by modern alternators, we may safely say that in no practical case can the distortion be detected by its introducing errors into the measurement of either emf, current or power, even with the most accurate instruments available.

It also follows that there is no appreciable difference in the ratio of transformation whether it be defined as the ratio of the mean effective values of the currents, or as the ratio of the primary to the undistorted part of the secondary; and hence there can be no objection against any of the null methods now in use, whether the detector be an electrodynamometer, an electrometer, or a rotating commutator with a direct-current galvanometer.

\section{EFFECT OF WAVE FORM UPON RATIO AND PHASE ANGLE}

While, as we have seen, no appreciable distortion is introduced by the transformer, the wave form may change the ratio appreciably. This has been pointed out by Rosa and Lloyd, ${ }^{18}$ who made some measurements, the result of which showed that while small, the effect could be detected by the methods then in use, if the distortion was large.

In Fig. 29 are plotted the results of measurements of the ratio of transformer $J$, which is a high-grade instrument, with 5 different wave forms, and with 2 different secondary impedance loads. It should be noted that the vertical scale is greatly mag- 
nified so as to separate the curves, also that the impedance load for the upper curves is somewhat severe. The curves are very nearly parallel, and the ratio at the smaller impedance load even with a wave distorted by 20 per cent of third harmonic differs only 0.05 per cent either way from the value with a sine curve, while with the large impedance in the secondary the corresponding change is only o.r 5 per cent and these are only half as great at ro per cent distortion. At 60 cycles the corresponding figures
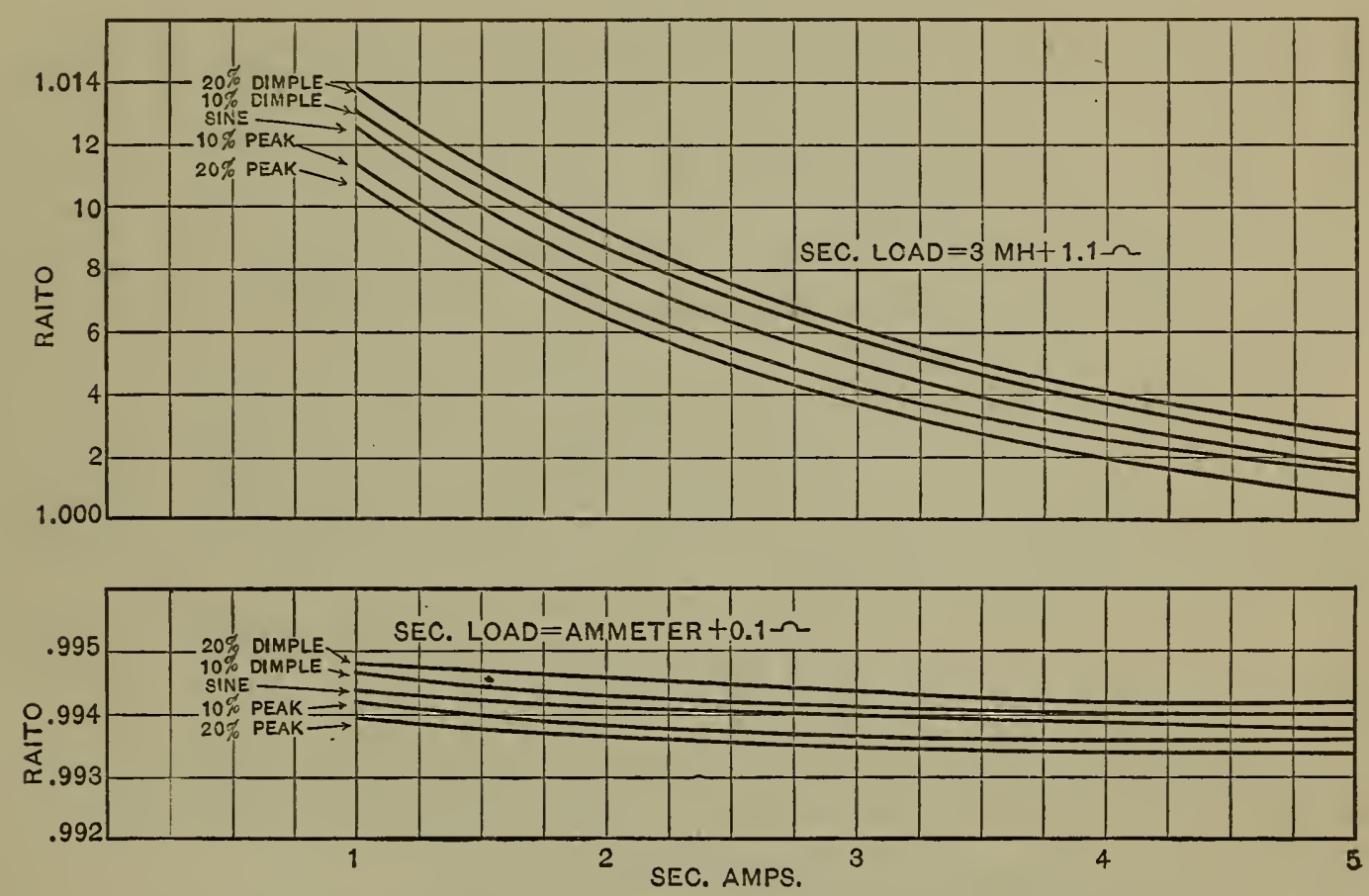

Fig. 29.-Effect of wave form upon ratio. Transformer J, 25 cycles.

would be considerably less. The relative accuracy of the measurements was 0.02 per cent.

The dimpled wave raises the ratio and the peak wave lowers it, which is in the direction that theory would predict, since a dimpled wave increases the iron losses and a peaked wave decreases them for the same effective value of emf, as has been shown by Lloyd ${ }^{19}$ and others.

The effect of wave form on the phase angle is very small. For example with 25 cycles, I.I ohm resistance in the secondary cir-

${ }^{19}$ This Bulletin, 5, p. 38I, I909, Reprint No. Io6.

$83226^{\circ}-\mathrm{II}-\mathrm{II}$ 
cuit, full load current, the following values of the phase angle were determined.

\begin{tabular}{l|c}
\hline Wave form & Phase angle \\
\hline $20 \%$ peak & $33.8^{\prime}$ \\
sine & $35.0^{\prime}$ \\
$20 \%$ dimple & $35.6^{\prime \prime}$ \\
\hline
\end{tabular}

About the same differences persisted over the current range, as near as could be told; that is, half a minute to a minute. The sensibility was proportionately less at the lower currents. Again, the direction of the change found is the same as would be predicted from theory. It is too small to be of any practical significance. With Io per cent distortion the effect was too small to be detected with certainty.

The effect of wave form upon ratio and phase angle is very similar to that of small changes in frequency, as might well be expected, and with good transformers is entirely negligible for the wave forms met in practice.

\section{SUMMARY}

I. While the ratio of transformation of current transformers usually decreases with increasing current, it may increase in individual cases, or even pass through a maximum.

2. The ratio and the phase angle performance may be accurately computed from the magnetic data of the core.

3. In general the slope of the ratio curve may be qualitatively predicted from the value of the Steinmetz exponent if the latter be assumed to be constant. But the iron losses, particularly at the low flux densities used, depart too widely from such a simple law for accurate work.

4. The slope of the ratio curve may be accurately computed from the slope of the curve obtained by plotting the core loss against the flux on logarithmic coordinate paper.

5. It is proposed that this logarithmic slope, or logarithmic derivative shall be called the ratio of variation. It is much more useful than the actual exponent. 
6. The methods now in use for determining the "exponent" fail to give a true exponent that will satisfy the equation $W=K B^{z}$, unless $z$ is a constant. The quantity actually determined by these methods is the ratio of variation.

7. The wave form of the secondary of a current transformer may be considered to be the same as that of the primary current for even the most precise measurements, as the distortion within the transformer is entirely negligible.

8. While the effect of variations in wave form on ratio and phase angle may be detected by accurate measurements, it is too small to be of practical importance, being of the same order of magnitude as the effect of small changes in frequency.

9. The null methods used for accurate determinations of ratio and phase angle all give the theoretically correct results, well within the experimental error, so that the accuracy attainable is decidedly greater than is required in practice.

\section{BIBLIOGRAPHY}

The following list is not intended as a complete bibliography of the current transformer, but it is believed that all the more important papers are included:

I. CAMPBELL, A.: On the measurement of very large and very small alternating currents.

Phil. Mag., 52, 27I; I896.

2. SchrotTkE, F.: Ueber Drehfeldmessgeräthe.

Elektrotechnische Zeitschrift, 22, p. 657; I901. (Current transformers are discussed on p. 665.)

3. WILDER, E. L.: Operation of the series transformer.

Elec. Journal, 1, p. 45I; I904.

4. CuRTis, K. L.: The current transformer.

Proc. Amer. Inst. Elec. Eng., 25, p. 7 I5; 1906.

5. DRySdal,i, C. V.: Some measurements on phase displacements in resistances and transformers.

Electrician, pp. 726 and 783 ; 1906.

6. MAKowER, A. J.: Measurement of phase difference. Electrician, 58, p. 695; 1907 .

7. HARRAR, E. S.: The series transformer.

Elec. World, 51, p. I044; r908.

8. Drysdale, C. V.: The use of shunts and transformers with alternating current measuring instruments.

Phil. Mag., 21, p. I36; I908.

9. Edgcumbe, K.: Industrial measuring instruments, p. I34.

New York; rgo8. (D. Van Nostrand Co.) 
IO. LLOYD, M. G.: What is the ratio of a transformer?

Elec. World, 52, p. 77; rgo8.

II. RosA, E. B. and M. G. LLOYD: The determination of the ratio of transformation and of the phase relations in transformers.

Bull. Bur. Standards, 6, p. r; rgog.

I2. Agnew, P. G. and T. T. Fitch: The determination of the constants of instrument transformers.

Bull. Bur. Standards, 6, p. 28I; 1909, Reprint No. $x_{3} 0$.

Elec. World, 54, p. 1042; I909.

I3. ORLICH, E.: Über die Anwendung des Quadranten-electrometers zu Wechselstrommessingen.

Electrotechnische Zeitschrift, 30, pp. 435, 466; 1909. (A discussion of the measurement of the constants of the current transformer is given on p. 468.)

I4. Robinson, L. T.: Electrical measurements on circuits requiring current and potential transformers.

Trans. Amer. Inst. Elec. Eng., 28, p. r005; rg09. (Discussion, p. 1040.)

I5. TовEY, H. W.: Recent developments in transforming apparatus.

Filectrician, 63, p. 659; I909.

I6. WERnicke, G.: Elektrotechnische Messungen und Messinstrumente, p. 59.

Braunschweig; rgo9. (Vieweg u. Sohn.)

I7. STERZEL, K. A.: Stromwandler fur Wechselstrom-Leistungsmessungen.

Electrotechnische Zeitschrift, 30, p. 489; 1909.

I8. GENKIN, W.: Sur les transformateurs d'intensité.

Lumière Électrique, 8, p. 67 ; I909.

I9. Young, A. P.: Theory and design of the current transformer.

Jour. Inst. E. E., 45, p. 670; rgro.

20. CAMPBElL, A.: On the use of mutual inductometers.

Proc. Phys. Soc. London, 22, 207; r9ro. (Section on tests of current transformers, p. 217.)

2i. Barbagfilata, A.: Prova indiretta dei transformatori di misura per forti intensità di correnti.

Atti Assoc. Elettr. Ital., 14, 639; r910.

22. Edgcumbe, K.: Notes on instrument transformers.

Elec. Rev. (Lond.), 67, p. 163; 1910.

23. Laws, F. A.: Determination of the constants of instrument transformers.

Elec. World, 55, p. 223; I9ro.

24. Sirarp, C. H. and Crawford, W. W.: Some recent developments in exact alternating current measurements.

Proc. Amer. Inst. Elec. Eng., 29, p. I207; 1910. (Current transformer ratios are treated on pp. I2II-I220.)

25. BAKER, H. S.: Current-ratio and pliase-angle test of series transformers.

Eilec. World, 57, p. 234; Jan. 26, rgrx.

Washington, April 6, I911. 